OPEN ACCESS

Edited by:

Kun Zhang,

Tongji University, China

Reviewed by:

Yanfen Cui,

Shanxi Provincial Cancer Hospital,

China

Qiufeng Zhao,

Shanghai University of Traditional

Chinese Medicine, China

Xiao-Yan Zhang,

Beijing Cancer Hospital, China

${ }^{*}$ Correspondence:

Weijun Peng

cjr.pengweijun@vip.163.com

Tong Tong

t983352@126.com

Specialty section: This article was submitted to

Radiation Oncology,

a section of the journal

Frontiers in Oncology

Received: 23 January 2022

Accepted: 04 February 2022

Published: 01 March 2022

Citation:

Hu F, Hu Y, Wang D, Ma X, Yue Y, Tang W, Liu W, Wu P, Peng W and Tong $T$ (2022) Cystic Neoplasms of the Pancreas: Differential Diagnosis and Radiology Correlation.

Front. Oncol. 12:860740. doi: 10.3389/fonc.2022.860740

\section{Cystic Neoplasms of the Pancreas: Differential Diagnosis and Radiology Correlation}

\author{
Feixiang $\mathrm{Hu}^{1}$, Yue $\mathrm{Hu}^{2}$, Dan Wang ${ }^{3}$, Xiaowen $\mathrm{Ma}^{1}$, Yali Yue ${ }^{4}$, Wei Tang ${ }^{1}$, Wei Liu ${ }^{1}$, \\ Puye $W^{5}{ }^{5}$, Weijun Peng ${ }^{1 *}$ and Tong Tong ${ }^{1 *}$ \\ 1 Department of Radiology, Fudan University Shanghai Cancer Center, Department of Oncology, Shanghai Medical College, \\ Fudan University, Shanghai, China, ${ }^{2}$ Hefei Cancer Hospital, Chinese Academy of Sciences (CAS), Hefei, China, ${ }^{3}$ Shanghai \\ Municipal Hospital of Traditional Chinese Medicine, Shanghai, China, ${ }^{4}$ Children's Hospital, Fudan University, Shanghai, China, \\ ${ }^{5}$ General Electric (GE) Healthcare, Shanghai, China
}

Although the probability of pancreatic cystic neoplasms (PCNs) being detected is raising year by year, their differential diagnosis and individualized treatment are still a challenge in clinical work. PCNs are tumors containing cystic components with different biological behaviors, and their clinical manifestations, epidemiology, imaging features, and malignant risks are different. Some are benign [e.g., serous cystic neoplasms (SCNs)], with a barely possible that turning into malignant, while others display a low or higher malignant risk [e.g., solid pseudopapillary neoplasms (SPNs), intraductal papillary mucinous neoplasms (IPMNs), and mucinous cystic neoplasms (MCNs)]. PCN management should concentrate on preventing the progression of malignant tumors while preventing complications caused by unnecessary surgical intervention. Clinically, various advanced imaging equipment are usually combined to obtain a more reliable preoperative diagnosis. The challenge for clinicians and radiologists is how to accurately diagnose PCNs before surgery so that corresponding surgical methods and follow-up strategies can be developed or not, as appropriate. The objective of this review is to sum up the clinical features, imaging findings and management of the most common PCNs according to the classic literature and latest guidelines.

Keywords: pancreatic cystic neoplasms (PCNs), radiology, intraductal papillary mucinous neoplasms (IPMNs), mucinous cystic neoplasms (MCNs), solid pseudopapillary neoplasms (SPN), serous cystic neoplasms (SCN)

\section{INTRODUCTION}

In recent years, the diagnostic rate of PCNs has risen and still keeps an increasing trend. Most patients have no clinical symptoms, and many are found incidentally. Because the etiology and malignant potential of PCNs are often not very clear, diagnosis and management of these neoplasms are challenging. Except for the tail, most of the pancreas is located outside the peritoneum on the posterior wall of the abdominal cavity. Early detection of PCNs is difficult in the absence of clinical symptoms, due to its deep position; most of them are detected incidentally on cross-sectional imaging, and these patients do not have typical pancreatic symptoms (i.e., pancreatitis, jaundice, and new-onset diabetes) (1). It is estimated that approximately $2 \%$ to $49 \%$ of routine imaging 
examinations such as computed tomography (CT) or magnetic resonance imaging (MRI) incidentally detect pancreatic cystic lesions, and this proportion increases with age (2).

The most common PCNs are IPMN, MCN, SCN and SPN. Based on epidemiological data, the incidence of MCN, SCN, and SPN in women is significantly higher than that in men, accounting for approximately $95 \%, 70 \%$, and $80 \%$, respectively, while IPMN is similar or slightly higher than that in men, approximately 55\% (3). In recent years, people's consciousness of these neoplasms has increased, partly due to the application of high-resolution CT/MR imaging. Although the awareness environment of the natural history and most appropriate treatment of various types of cystic lesions is gradually ameliorating, the diagnosis and treatment of PCNs are still challenging. They are usually composed of different solid components, and each solid component exhibits divergent biological behavior, occurring from benign to borderline or even malignant (4). Due to the partial overlap of benign and malignant imaging features, the differential diagnosis of benign versus malignant PCNs is relatively difficult. Moreover, in view of the potential malignant transformation of some benign tumors, the "silent epidemic" of symptomless PCNs has created a real predicament in the treatment and management strategies of such patients.

Different PCNs have relatively specific imaging manifestations, so we can not only identify these tumors by morphological features but also objectively and quantitatively analyze the tumor phenotype by radiomics. Radiomics features have broad application prospects in differential diagnosis, prognosis and efficacy evaluation of PCNs (5). Combined with clinical manifestations and imaging features, radiomics provides an opportunity for the preoperative accurate diagnosis of pancreatic cystic tumors. This review will focus on the clinical features and typical imaging manifestations of different types of PCNs and discusses the latest radiomics research. Thus, it provides an important reference for the precise preoperative diagnosis and individualized management of PCNs. A comprehensive discussion of nonneoplastic pancreatic cystic lesions (PCLs) are not included in this review.

\section{THE MAJOR PANCREATIC CYSTIC NEOPLASMS}

The most common types of PCNs (Figure 1) are mucinproducing intraductal papillary mucinous neoplasms (IPMNs, encompassing branch-duct IPMNs, main-duct IPMNs and mixed-type IPMNs) and mucinous cystic neoplasms (MCNs). Less common subtypes include nonmucinous tumors such as solid pseudopapillary neoplasms (SPNs) and serous cystic neoplasms (SCNs) (6). The incidence of PCNs varies with the population distribution. For example, IPMNs approximately $21 \%$ to $33 \%$, MCNs account for $10 \%$ to $45 \%$, SPNs account for less than $10 \%$, and SCNs account for $32 \%$ to $39 \%$ of all PCNs in the Western Hemisphere. There was a national survey report from Korea shows that IPMNs approximately $41 \%$, MCNs approximately $25.2 \%$, SPNs approximately $18.3 \%$, SCNs approximately $15.2 \%$, and others account for $0.3 \%$ of PCNs $(7$, 8). However, the actual incidence of various types of PCN is unknown. The estimated relative frequencies of PCNs removal from one center of 14,424 patients that treated with surgery over a 15-year period were as detailed below: MD-IPMN, BD-IPMN, MCN, SCA, and SPN account for 25\%, 26\%,11-18\%,13-23\% and 4-7\%, respectively (9). PCNs often do not have typical clinical symptoms. An Italian multicenter prospective study of pancreatic cystic tumors showed that 338 of 1370 cases (24.7\%) had one or more clinical symptoms: abdominal pain $(214,15.6 \%)$, acute pancreatitis $(106,7.7 \%)$, diarrhea $(12,0.9 \%)$, gallstones $(39,2.8 \%)$, weight loss $(21,1.5 \%)$, fatigue $(9,0.7 \%)$, loss of appetite $(2,0.1 \%)$, diabetes $(40,2.9 \%)$ and jaundice $(14,10 \%)$. However, most patients were asymptomatic (1036, $75.6 \%)(10)$. The specific characteristics of the four most common PCNs are exhibition in Table 1.

\section{Intraductal Papillary Mucinous Neoplasm}

IPMN is a benign, borderline, low-grade dysplasia or invasive cancer derived from pancreatic ductal epithelium. Tumor cells are tall columnar mucous-rich epithelial cells, with or without papillary protrusions, extensively invading the main pancreatic duct (MPD) and/or branch pancreatic duct (BPD), causing cystic dilation. With the continuous development of diagnostic standardization and imaging techniques, IPMNs are becoming increasingly routinely diagnosed in clinical practice. According to the communication with the pancreatic duct, they are morphologically divided into MD-IPMN, BD-IPMN and MTIPMN. Approximately $40 \%$ to $65 \%$ of IPMNs occur in the branch pancreatic ducts, while they are found in the MPD accounting for about $15 \%$ to $35 \%$ cases. The probability of simultaneous occurrence in the both pancreatic ducts is only 15\%-20\% (28).

According to the different histology and mucin expression of IPMN, it can be divided into four epithelial subtypes as below: gastric-type, intestinal-type, pancreatobiliary-type and oncocytic-type, each of which has various kinds of risks of malignant progression. Oncocytic- and gastric-type IPMNs are often low-grade neoplasms, while intestinal- and pancreatobiliary-type IPMNs have a disposition to high-grade neoplasms and are usually related to invasive cancer (IC) (11). The prognosis of IPMNs is superior to pancreatic ductal adenocarcinoma (PDAC) after surgical resection (29). It has been reported that the incidence of cancers derived from IPMN is between $6 \%$ and $46 \%(30-33)$, including IPMN with highgrade dysplasia (HGD) and IC, and its prognosis is as poor as that of PDAC (34). Assessing the rate of malignancy in IPMN has become a clinical challenge. The risk factors for malignant tumors include weight loss, patient age, relationship with mural nodules, increased jaundice/bilirubin levels, and elevated CEA levels. However, there is no established standard that can safely and accurately distinguish malignant and nonmalignant lesions (35). Therefore, the key to the treatment of IPMN is to accurately predict the risk of malignancy. At the same time, it is also important to evaluate the probability of surgical resection 


\section{a: hyperplasia \\ b: adenoma \\ c: adenocarcinoma \\ d: mucus \\ e: mural nodule \\ f: cystic cavity}
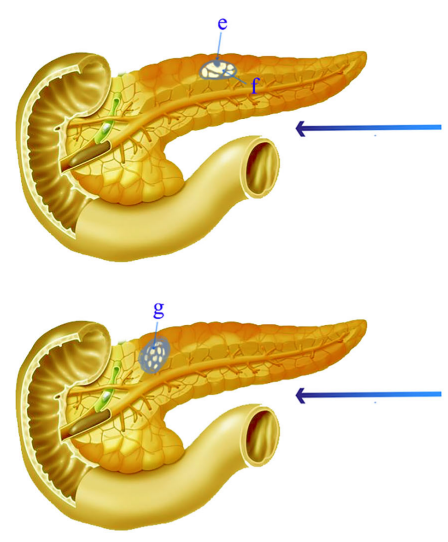

$\mathrm{MCN}$

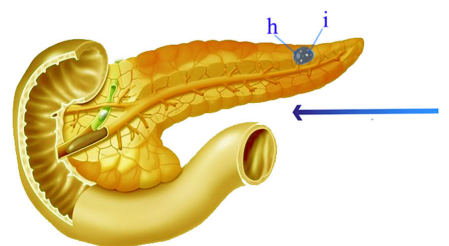

g: septum

h: hemorrhage

i: calcification

\section{Pancreatic Cystic Neoplasms, PCNs}

FIGURE 1 | Schematic representation of the characteristic morphological and imaging features of various PCNs.

benefit. Imaging takes a significant role in the evaluation and detection of IPMNs (36).

The aims of imaging examination of IPMN are as follows: first, to detect IPMN and exclude other PCLs; second, to distinguish the relationship between lesions and the pancreatic duct, which is conducive to typing; and third, to determine the key risk factor of malignancy and estimate the resectability of clinical surgery. Various imaging methods are used to achieve these goals.

\section{Mucinous Cystic Neoplasm}

MCNs are cystic tumors derived from the pancreatic epithelium that have the potential for malignancy. They are relatively rare pancreatic cystic tumors in the clinic, accounting for $29 \%$ of all PCNs (37). Compared with IPMNs, MCNs do neither communication with the MPD nor BPD system. They are often solitary and are covered by a thin fibrous cyst wall. The cyst is lined with tall columnar mucous cells that secrete mucin and often form papillae, and the subepithelial stroma is often ovarian-like stroma with abundant cells (38). There is no significant difference in incidence between sexes in their $60 \mathrm{~s}$ and 70s, and the probability of occurrence in the body/tail of the pancreas is greater than that in the pancreatic head $(67.3 \%$ $99.4 \%$ ) (39), with $89.5 \%$ in the present series.

The malignant probability of MCNs varies between $6 \%$ and $36 \%$, which is still significant (12). The features predictive of malignancy include irregular or focal thickening of the cyst wall, a large volume, and solid content inside or outside the cyst (40, $41)$. The size $(>8.5 \mathrm{~cm})$ and volume of the $\mathrm{MCNs}$ on $\mathrm{CT} / \mathrm{MR}$ imaging are the only features associated with HGD/IC, and the average growth rate is very slow, about 4 millimeters ( 0.16 in) every year (42). The mucinous transitional epithelium is the origin of almost whole malignancies arising from MCNs. MCNs can be divided into three major categories according to the grade of dysplasia as well as IPMNs: low- or intermediate-GD, HGD and IC $(13,43)$. Resection is advocated for whole types of MCNs 
TABLE 1 | The clinical and image features of common pancreatic cystic neoplasms $(4,10-27)$.

\begin{tabular}{|c|c|c|c|c|}
\hline Characteristics & IPMN & MCN & SCN & SPN \\
\hline Age (decade) & 5th-7th & 4th-5th & 5th-6th & 2-3th \\
\hline $\begin{array}{l}\text { Gender } \\
\text { distribution }\end{array}$ & Equal & $90-95 \%$ female & $70 \%$ female & $90 \%$ female \\
\hline Location & Common in pancreatic head & Body and tail & Entire pancreas & $\begin{array}{l}\text { Throughout, common in body and } \\
\text { tail }\end{array}$ \\
\hline Imaging features & $\begin{array}{l}\text { Multiple mural nodules, pancreatic } \\
\text { duct dilatation, ductal } \\
\text { communication, cyst or cluster of } \\
\text { cysts }\end{array}$ & $\begin{array}{l}\text { Large cysts with thick septae, } \\
\text { peripheral calcification, mural } \\
\text { nodules }\end{array}$ & $\begin{array}{l}\text { Star-shaped central scar with } \\
\text { calcification, microcystic multiple } \\
\text { small cyst, sometimes oligocytic }\end{array}$ & $\begin{array}{l}\text { Local capsule interruption, cystic } \\
\text { degeneration, calcification and } \\
\text { hemorrhage, floating cloud sign }\end{array}$ \\
\hline Cyst fluid & Viscous, mucin-rich & Viscous, mucin-rich & Thin & Bloody \\
\hline Classification & MD-IPMN, BD-IPMN, MT-IPMN & $\begin{array}{l}\text { Low- or intermediate-grade } \\
\text { dysplasia, high-grade dysplasia, an } \\
\text { associated invasive carcinoma }\end{array}$ & SMA, SOA, SSCA & Low malignant neoplasm \\
\hline $\begin{array}{l}\text { Clinical } \\
\text { symptoms }\end{array}$ & $\begin{array}{l}\text { Incidental finding, jaundice, } \\
\text { pancreatitis, malignancy-related }\end{array}$ & $\begin{array}{l}\text { Incidental finding, abdominal pain, } \\
\text { malignancy-related }\end{array}$ & $\begin{array}{l}\text { Incidental finding, abdominal pain, } \\
\text { mass effect }\end{array}$ & $\begin{array}{l}\text { Incidental finding, abdominal pain, } \\
\text { mass effect }\end{array}$ \\
\hline $\begin{array}{l}\text { Connection with } \\
\text { MPD }\end{array}$ & Yes & No & No & No \\
\hline $\begin{array}{l}\text { Solitary or } \\
\text { multifocal }\end{array}$ & Solitary/multifocal & Solitary & Solitary & Solitary \\
\hline CEA & z192 200ng/ml (80\%) & z192 200ng/ml (80\%) & $<5 \mathrm{ng} / \mathrm{ml}$ & Unknown \\
\hline Amylase & High & Low & Low & Low \\
\hline $\begin{array}{l}\text { Molecular } \\
\text { markers }\end{array}$ & $\begin{array}{l}\text { KRAS mutation(+) (80\%), GNAS } \\
\text { mutation(+) (41-66\%) }\end{array}$ & $\begin{array}{l}\text { KRAS mutation(+) (50\%-75\%), } \\
\text { GNAS mutation(-), CTNNB1 } \\
\text { mutation(-) }\end{array}$ & $\begin{array}{l}\text { VHL, VEGF-A>8500pg/mL, VEGF- } \\
\text { C>200pg/mL, MUC1, MUC6 }\end{array}$ & $\begin{array}{l}\text { CTNNB1, B-catenin, LEF1, TFE3S, } \\
\text { SOX11 }\end{array}$ \\
\hline Cytology & $\begin{array}{l}\text { Columnar cells, +mucin, variable } \\
\text { atypia }\end{array}$ & $\begin{array}{l}\text { Columnar cells, +mucin, variable } \\
\text { atypia }\end{array}$ & $\begin{array}{l}\text { Often acellular or cuboidal cells } \\
\text { stain, +glycogen }\end{array}$ & $\begin{array}{l}\text { Branching papillae with myxoid } \\
\text { stroma }\end{array}$ \\
\hline $\begin{array}{l}\text { Malignant } \\
\text { probability }\end{array}$ & Medium or high & Medium & Negligible & Low or medium \\
\hline
\end{tabular}

IPMN, intraductal papillary mucinous neoplasms; MCN, Mucinous Cystic Neoplasm; SCN, Serous Cystic Neoplasm; SPN, solid pseudopapillary neoplasms; MPD, main pancreatic duct; MD-IPMN, main-duct IPMN; BD-IPMN, branch-duct IPMN; MT-IPMN, mixed-type IPMN; SMA, serous microcystic adenoma; SOA, serous oligocystic adenoma; SSCA, solid serous cystadenomas.

according to current the guidelines and clinical consensus unless there are contraindications to surgery (44). For MCNs with IC, the prognosis is closely related to the extent of lesions invasion, tumor stage and $\mathrm{R} 0$ resection rate. The two- and five-years survival rates of resectable MCNs with IC are $67 \%$ and $50 \%$, respectively (7). Therefore, early detection and identification of MCNs with invasive cancer by imaging methods are of great significance.

\section{Solid Pseudopapillary Neoplasm}

SPNs are an rare pancreatic tumor and, as their name implies, have a solid pseudopapillary structure formed by epithelial cells of a single shape in a loose arrangement. They are prone to hemorrhage and cystic transformation. These tumors account for only $0.2 \sim 2.7 \%$ of all exocrine pancreatic neoplasms (45). According to the morphological and structural characteristics of the lesion, different reports use the nomenclature solid-cystic neoplasm, papillary-cystic neoplasm, solid-cystic acinar neoplasm, solid-papillary neoplasm, but the actual use of "solid pseudopapillary neoplasm" (SPN) is similarly only a descriptive name that represents morphological features yet retains the openness of histogenesis. Regardless of the presence or absence of metastatic disease, SPNs are generally considered to be lowgrade tumors with an indolent growth pattern. The origin of these tumor cells in the pancreas is uncertain. There are two classical theories about the origin of SPN: the one suggests that it origins from pluripotent pancreatic cell, then the other proposes a female genital bud origin (46).

Due to the wide application of high-quality and -resolution imaging examinations, mainly US, CT, and MRI, it has been reported high frequency in the past few decades. In a recent review of all SPN description that published in the English journal up to 2014, Law et al. (47) confirmed a total of 2744 cases of this neoplasm. Yao et al. systematically reviewed 2,450 SPN cases in a Chinese population before January 2020, which was published in both the Chinese and English literature (48). They concluded that SPN is an indolent neoplasm and seldom seen that mainly occurs in young females. The clinical manifestations are abdominal masses and abdominal pain, most of them present as pancreatic head and tail space occupying, and the prognosis is excellent after complete resection. Generally, they are indolent, but a few have malignant potential. Regrettably, the prognostic factors that predict malignant potential have been hard to identify (49). Most patients present with local lesions, and only 9-15\% have metastases or local infiltration.

At present, the main treatment is still surgical resection, and its prognosis is different from that of pancreatic cancer. According to reports, the five-year survival rate can be as high as $94-97 \%(45,50)$. Rare SPNs can occur at any age and in both genders, especially young females. Although the survival rate is typically high, histological images cannot accurately predict its biological behavior. Lesions without obvious malignant signs but only suspicious morphological signs can also cause late 
recurrence, metastasis and even death. The exact histogenesis is still unclear, and it may originate from primordial cells. More research on SPT is needs for further clarification.

\section{Serous Cystic Neoplasms}

SCNs are benign tumors of the pancreatic exocrine glands that account for $16 \% \sim 33.3 \%$ of whole cystic neoplasms of the pancreas. It is a slow-growing benign lesion with an extremely low probability of malignant transformation $(14,15,51)$. The concept of SCNs as benign disease entities without the risk of malignant transformation was revised after George et al. revealed the first case of a malignant pancreatic SCN in 1989 (52). The malignancy of SCNs, serous cystadenocarcinomas, are limited to $25 \sim 30$ cases report in the global literature, representing $<1 \%$ of all SCNs (15), including the largest sample size, which found three patients with serous cystadenocarcinomas among 2622 cases (53). Therefore, SCNs of the pancreas have extremely low malignant potential but are not totally benign.

Patients are often discovered with SCN in their late 50th or early 60th. SCN usually develops in the body/tail of the pancreas. Despite these neoplasms are mostly benign, they often grow slowly and may have large diameters (13). SCNs are representatively honeycombed microcystic tumors consisting of uniform, cuboidal, glycogen-rich epithelial cells. Thus far, there are four known variants of serous cystadenoma, namely, macrocystic serous cystadenoma, solid serous adenoma, VHL-related SCN, and mixed serous neuroendocrine neoplasm, in which the serous epithelial components of these variants are identical to those of serous cystadenoma.

Pancreatic serous cystadenomas are benign lesions and could be regulated by surveillance, which does not commonly mandate surgical resection unless they exhibit aggressiveness or unspecific characteristics that hinder accurate diagnosis. CT is the preferred first-line examination modality for characterizing SCNs and differentiating them from their mimickers (54).

\section{IMAGING DIAGNOSIS AND PRECISION IMAGING}

Multiple imaging modalities can help to further distinguish a PCN, facilitating the findings, characterization, and evaluation of the presence of aggressive behavior and the evaluation of resectability in patients with obviously malignancy. Imaging modalities have unique advantages and potential weaknesses in terms of PCN evaluation. Radiomics is an emerging field in quantitative imaging that uses techniques that advance imaging features to objectively and quantitatively investigate tumor phenotypes. Noninvasive medical imaging such as US, MRI, CT, and positron emission tomography (PET) can be used to assess tumor and anatomical tissue features for tumor management (55-57). Radiomics can obtain high-content information through identification, extraction, quantitation, and processing to identify imaging signatures or phenotypes.

Information from surrogate imaging biomarkers combined with multifarious demographic, biologic ("omic") and outcome-driven methods can be used to develop precision medicine strategies.
Accurate imaging biomarkers have been described in a large number of neoplasms. Medical images store more information than trained physicians can see; thus, more details about the region of interest that are embedded in this hidden information can be extracted and analyzed by computational tools than has been previously observed $(58,59)$. Manual identification of cyst type has an accuracy of only $60 \sim 70 \%$, even by well-trained radiologists (60). Therefore, the development of imaging markers using radiomics could increase the correct identification of the type and malignant rates of PCNs.

Regrettably, radiological research to assess the risk of PCN malignancies, especially IPMN, is very limited. In one of the earliest research, an algorithm that distinguishes between the four most common types of PCNs (IPMN, MCN, SCA, and SPN) was proposed by Dmitriev and his colleagues. They revealed an integrated model that combines patient demographic factors with intensity and shape characteristics extracted from cyst images. Segmentation of the cystic neoplasms was acquired by a semiautomated graph-based segmentation technique, at the same time, an random forest classifier and convolutional neural networks were applied for feature selection. This groundbreaking research acquired an accuracy of approximately $84 \%$ in distinguishing various types of cysts (61). A recent research revealed a computer-aided diagnosis (CAD) scheme based on radiomics and emphasized the role of radiomics analysis as a new noninvasive tool to improve the accuracy of the preoperative diagnosis of SCN (59). Another study showed that a comprehensive nomogram combining clinical characteristics and fusion radiomics features could identify SCNs from mucin-producing PCNs (58). With the in-depth study of radiomics methods in the field of tumors, it is believed that more research will focus on the differential diagnosis of PCNs in the future.

At present, advanced imaging techniques are increasingly utilized in clinical practice, and the detection rate of PCLs has started to increase gradually. For example, Laffan et al. reported that PCLs were detected in about $2.6 \%$ of items using multidetector computed tomography (MDCT) (62), suggesting that CT is the first available source of imaging data for diagnosis. One previous study revealed an accuracy of $67-70 \%$ for discriminating 130 pancreatic cysts on CT scans, which were performed by two readers with more than 10-years of experience in abdominal diagnosis (60). In addition, the accuracy of MDCT for characterizing PCN ranges from 56\% to $85 \%$ (63), and the wide availability, high spatial resolution, and rapidity of acquisition make MDCT ideal for the initial PCN assessment (63). Furthermore, the presence of high-risk stigmata, including a solid component or mass within the cyst, or the presence of mural nodules can be identified by CT imaging. However, MDCT also has disadvantages. It is still difficult to characterize the histopathologic subtype of PCN, as their CT features overlap (64). Besides, the ionizing radiation inherent to CT might result in suboptimal use effects, especially for continued followup examinations.

The raising availability and use of dual-energy CT scanners may be advantageous to reduce the overall dose to the patient, thus decreasing the number of acquired phases by using virtual 
unenhanced imaging. Although CT spectral imaging can provide additional information and multiparametric analysis can achieve greater results than single-parameter analysis in differentiating serous and mucinous content, it is difficult to combine multiparametric analysis and CT spectral imagingderived quantitative parameters to improve the diagnostic performance (65).

Given the extensive use of high-quality and -resolution MDCT, recent studies have assessed advanced computer-based quantitative image analysis to obtain additional information for identifying characteristics that might be helpful to predict high-risk IPMN. Hanania et al. evaluated 53 cases with IPMN and distinguished 14 imaging characteristics (biomarkers) to differentiate between LGD and HGD in IPMN. Using the top 10 of the 14 biomarkers, an AUC of 0.96 was achieved, with a sensitivity of $97 \%$ and specificity of $88 \%$ (66). The results of this study indicated that HGD/IC IPMNs have distinct radiomics features that could be utilized to stratify patients via noninvasive imaging. Permuth et al. also distinguished malignant from benign IPMN by using radiomics with 14 radiologic features in 38 cases; however, they integrated 5-miRNA data and achieved an AUC of 0.92 , with a sensitivity of $83 \%$ and specificity of $89 \%$ (67). Such high sensitivity and specificity are conducive to improving the clinical discrimination ability of benign and malignant IPMNs so that targeted and individualized treatment strategies can be adopted.

Yang et al. (68) identified 25 patients with $\mathrm{MCN}$ from 53 patients with SCN using a preliminary model based on texture features (GLCM, GLRLM, GLZLM, and NGLDM) extracted from contrast-enhanced CT images that were selected via LASSO regression and random forest classifiers. Fascinatingly, they also found a good correlation among the extracted texture features extracted from CT images of $2 \mathrm{~mm}$ and $5 \mathrm{~mm}$ thick slices, which had previously been neglected in many previous studies. Although the feature extraction was not affected by a difference in slice thickness, they suggested using CT images with similar slice thicknesses for radiomics analysis. They acquired an accuracy of $74 \%$ in the slice thickness of $2 \mathrm{~mm}$ group and $83 \%$ in the slice thickness of $5 \mathrm{~mm}$ group in the validation group.

The proposed radiomics-based CAD scheme could increase the accuracy of the preoperative diagnosis of pancreatic serous cystic neoplasms, showing an AUC of 0.767 in the crossvalidation group and 0.837 in the independent validation group (59). It has also been suggested that using CT alone is of limited value in differentiating between serous and mucinous lesions (69). The study showed that PCNs displaying central scarring, central calcification or the circumvascular sign on CT could be diagnosed as SCAs. When either of the first two features is combined with the circumvascular sign, the diagnostic sensitivity could be improved (14). The malignant probability of SCN is significantly lower than that of MCN. Thus, a followup observation strategy can be used for some patients.

The reported incidence of detecting asymptomatic pancreatic cysts on MRI is about 15\% (70). The prevalence on MRI is higher overall, ranging from $2.4 \%$ to $20 \%$, and increases with age to approximately $40 \%$ in patients older than $70 \mathrm{y}(71,72) . \mathrm{T}_{2} \mathrm{WI}$ are exquisitely sensitive to fluid-filled structures. Thus, small parenchymal pancreatic cysts or the MPD/BPD system with MRCP can be visualized by $\mathrm{T}_{2}$-weighted $\mathrm{MRI}$, which is the primary MRI pulse sequence (73). Compared to MDCT, MRI is more sensitive overall for detecting small pancreatic cysts $<3$ $\mathrm{cm}$ (74). Furthermore, MRI can detect more PCLs smaller than $20 \mathrm{~mm}$ than CT. For lesions larger than or equal to $20 \mathrm{~mm}$, MRI can depict a greater level of internal details than CT, which could aid clinicians in making management decisions (75). Hoffman et al. (76) demonstrated that entropy could be prognostic for malignancy by extracting a few intensity histogram-based statistical features from MR images of 18 patients with BDIPMN. In a concept-of-proof study involving 38 patients, there were 20 benign and 18 malignant IPMNs. MRI/MRCP has an additional advantage over MDCT for patients who require repeated imaging for follow-up because of the lack of radiation exposure. Disadvantages of MRI include its lower spatial resolution, low sensitivity to detect calcifications, and motionrelated artifacts.

\section{The CT and MR Manifestations of IPMN}

Among the three types of IPMN, the branched type is the most common, followed by the mixed type, and the MPD type is relatively rare. The latter is further divided into two sub-types, the segmental type and the diffuse type. The communication between cystic lesions and the MPD is one of the diagnostic points of branchtype IPMN. Branch-type IPMN specific imaging findings are as follows: tubular or earthworm-like shadows in low-density cystic lesions, cystic walls and septate microenhancing nodules. The dilated MPD is not limited to the distal end of the lesion. The imaging signs that affect the diagnosis are as follows: the lesions are oval or dumbbell-shaped, the lesions' density is close to that of water, and there are non-separations or tiny nodules in the lesions. Thin-slice scanning combined with three-phase enhancement, coronal or sagittal image reconstruction, and careful observation of cyst wall and intralesional structures can help improve the diagnostic accuracy (Figure 2).

The MD-type IPMN-specific imaging manifestations are as below: moderate or greater dilatation of the MPD, continuous expansion of the pancreatic duct without bead-like changes, enhanced mural nodules on the cyst wall, slight atrophy of the pancreatic parenchyma, and markedly dilated MPD asymmetry with mildly atrophied pancreatic parenchyma (Figure 3). Imaging signs that affect the diagnosis are as follows: in the early stage of the disease, dilatation of the MPD is limited or mild. In the early stage of the disease, this is easily confused with the slight dilatation of the pancreatic duct caused by anatomical variations; diffuse IPMN is easily confused with chronic pancreatitis, and localized IPMN is easily confused with pancreatic fusiform pseudocysts.

Mixed-type IPMN often comprises both branched IPMN and MPD IPMN, but it is not a simple combination of the two. In MT-IPMN, the expansion of the MPD can be localized or diffuse, and localized expansion can manifest as multiple discrete segmental expansions, but no beaded changes occur. There is no strict boundary between the limited MPD dilatation and branched IPMN with mild MPD dilatation. Some studies believe 

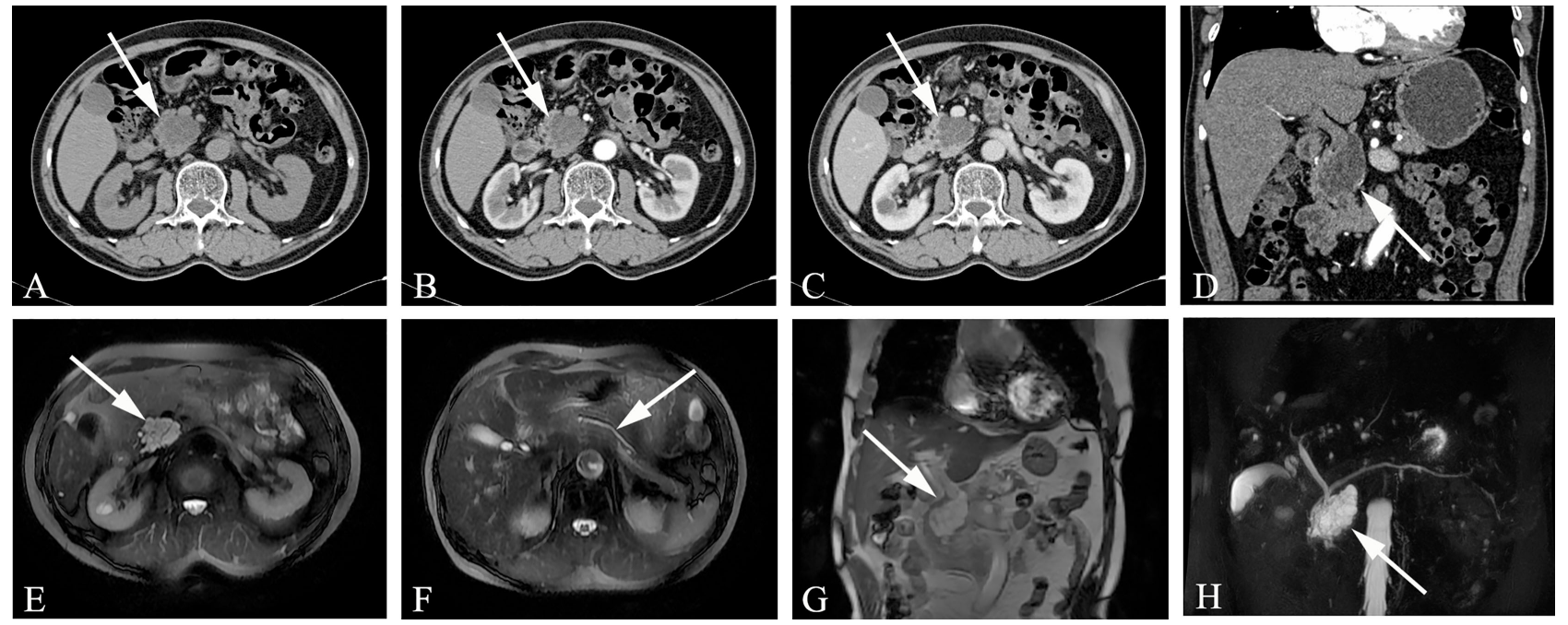

FIGURE 2 | Branch-type IPMN (Male, 66y, physical examination revealed a pancreatic mass for a week). (A-C) The CT plain scan, arterial phase and venous phase at the same level; (D) Coronal image in arterial phase. (E) $\mathrm{T}_{2}$-weighted cross-sectional image; (F) $\mathrm{T}_{2}$-weighted cross-sectional image at another level of the same patient; (G). $T_{2}$-weighted coronal image; H. MRCP reconstruction map; (A) The CT plain scan showed multilocular cystic mass of pancreatic head with septation and clear boundary. The density is slightly higher than that of water. (B-D) Contrast enhanced scan showed moderate enhancement of mural nodules in the dilated branch pancreatic duct. (E-H) Septa can be seen in the lesion and the MPD was slightly dilated. The white arrows in the Figures only indicate the location of the neoplasm.
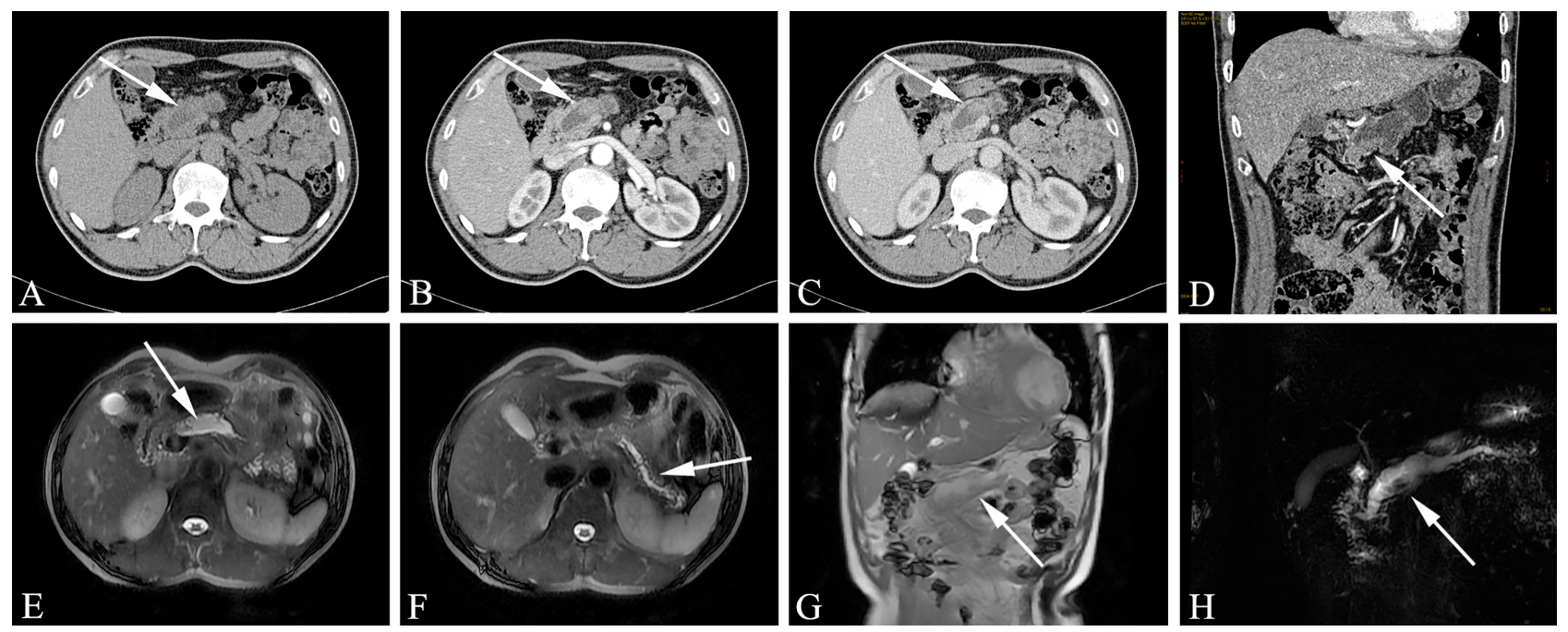

FIGURE 3 | Main-duct IPMN (Male, 57y, abdomen pain for one month). The sequence distribution of images is the same as that in Figure 2. (A) The CT plain scan showed nodule in the neck of the pancreas, with a CT value of about $31 \mathrm{HU}$. The MPD is obviously dilated, the pancreatic parenchyma is slightly atrophied, the dilated pancreatic duct is low-density, and the density is similar to that of water. (B, D) In the arterial phase, the nodule of the pancreatic neck was moderate enhanced, with a CT value of about $65 \mathrm{HU}$, and the dilated pancreatic duct showed more clearly. (C) In the venous phase, the nodule showed continuous enhancement, at this time, the CT value is $72 \mathrm{HU}$; (E-G) The $T_{2}$-weighted imaging shows pancreatic duct dilatation with multiple mural nodules. (H) MRCP shows the MPD dilated significantly throughout the whole pancreas. The white arrows in the Figures only indicate the location of the neoplasm.

that mixed IPMN is caused by the further development of branched IPMN, which is the key to distinguishing between branched IPMN and the mixed type. It is unclear whether there are tiny nodules in the MPD that are adjacent to the expansion, and if there are tiny nodules in the expanded MPD, it is of a mixed-type (Figure 4). Mixed-type IPMN can be accompanied by one or more branch types. Therefore, the imaging manifestations of mixed-type IPMN are more complex and can differ, but simultaneous expansion of the BPT and MPD is also the easiest subtype to diagnose. 

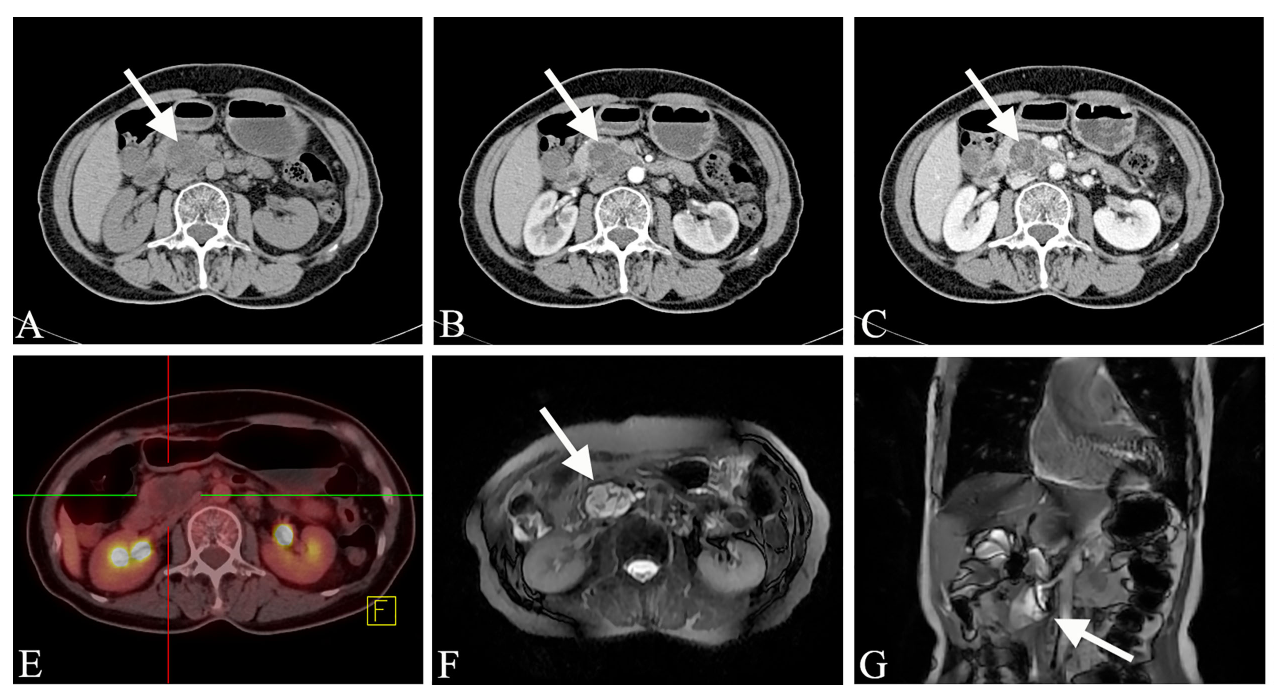

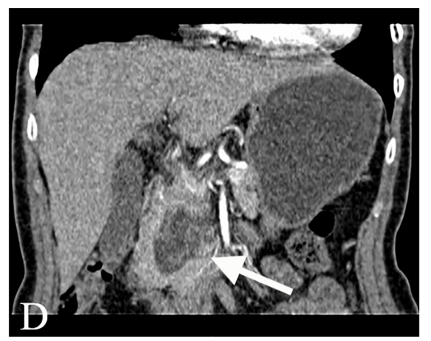

FIGURE 4 | Mixed-type IPMN (Female, 56y, abdomen pain for half year). (A-C) The CT plain scan, arterial phase and venous phase at the same level; (D) Coronal image in arterial phase. (E) PET cross-sectional image; (F) T2-weighted cross-sectional image; (G) $T_{2}$-weighted coronal image; (H) MRCP reconstruction map; (A) The CT plain scan showed multilocular cystic mass of pancreatic head with septation and clear boundary. The density is slightly higher than that of water. (B, C) Contrast enhanced scan showed moderate enhancement of mural nodules in the dilated MPD and branch pancreatic duct. (D) Diffuse dilatation of the MPD with enhanced mural nodules, which is the key to the diagnosis of mixed-type IPMN. (E) No obvious FDG uptake was found in the lesions by PET-CT. (F-H) Magnetic resonance imaging showed a multilocular cystic mass in the pancreatic head with multiple mural nodules, which communicated with the pancreatic duct. The white arrows in the Figures only indicate the location of the neoplasm.

Magnetic resonance $\mathrm{T}_{1} \mathrm{WI}$ showed that the liquid in the pancreatic duct of the IPMN dilated pancreatic duct had a low signal that was slightly higher than water; $\mathrm{T}_{2} \mathrm{WI}$ showed a high signal that was slightly lower than water. Some lesions showed hyperintensity on $\mathrm{T}_{1} \mathrm{WI}$ and hyperintensity on $\mathrm{T}_{2} \mathrm{WI}$. The spatial resolution of MRI is limited, and the ability to show small mural nodules is not as good as CT, but MR helps to show larger nodules. The nodules show a lower signal on $\mathrm{T}_{1} \mathrm{WI}$, which is between normal pancreatic tissue and dilated pancreatic duct fluid. $\mathrm{T}_{2} \mathrm{WI}$ is helpful to show streak-like hypointense separation or dilated pancreatic duct wall within the hyperintensity of branched IPMN, showing isohypointense mural nodules in the dilated pancreatic duct. The DWI signal of pancreatic IPMN varies greatly. Some DWI is isointense, and some is hyperintense. DWI helps to detect metastatic lymph nodes. MRCP helps to determine the relationship between the lesion and the MPD and shows the MPD protruding into the duodenum. Thin-layer MRCP is more helpful to determine the relationship between the MPD and the duodenum. During dynamic enhancement, the enhancement of the pancreatic duct wall of IPMN is similar to that of CT, the enhancement of small nodules is not as good as that of CT, and the enhancement of large nodules is close to or better than CT.

\section{The CT and MR Manifestations of MCN}

Mucinous cystadenoma with invasive carcinoma often manifests as multilocular cystic lesions with uneven wall thickness, wall nodules and calcifications in the lesion. After enhancement, the intracapsular septum thickens, and the wall nodules are obviously strengthened. Sometimes it is not easy to distinguish between benign and malignant tumors by imaging. If the following signs appear in the cyst, mucinous cystadenoma with invasive carcinoma is often indicated: (1)There are more solid components in the cyst. (2)There are obviously enhanced mural nodules. (3) Irregular thickening of the cyst wall and the presence of multiple daughter cyst near the large cyst. (4)Local pancreatic lymphadenopathy and intrahepatic metastasis are observed and adjacent large blood vessels have been invaded. (5The tumor is large, with a diameter $>8 \mathrm{~cm}$.

Mucinous cystadenoma usually manifests as a clear boundary with hypo-intensity on $\mathrm{T}_{1} \mathrm{WI}$ and hyper-intensity on $\mathrm{T}_{2} \mathrm{WI}$, but sometimes it has different manifestations due to the composition of the cyst fluid. The advantage of MRI is that it can accurately reflect the composition of the cyst fluid of mucinous cystadenoma. Sometimes the signal on $\mathrm{T}_{1} \mathrm{WI}$ is uneven and has a high signal, which is pathologically related to mucin in the cyst fluid or intracystic hemorrhage. In addition, MRI shows better separation between the wall and wall nodules in the lesion capsule than CT. The cyst cavity of mucinous cystadenoma is generally not connected to the pancreatic duct, which helps to distinguish it from intraductal papilloma (communicating with the pancreatic duct). MRCP examination is helpful for determining whether the mucinous cystadenoma is connected to the pancreatic duct. MR examination of mucinous cystadenoma with invasive carcinoma can not only show that the tumor is cystic but also clearly depicts the tumor cyst wall, septum and mural nodules (Figure 5).

Uneven thickening of the intratumoral septum and cyst wall or the appearance of mural nodules, invasion of the common bile duct (CBD) or pancreatic duct (PD) and surrounding blood vessels are all helpful for the diagnosis of mucinous cystadenoma with invasive carcinoma. MR examination is helpful for the differentiation of 

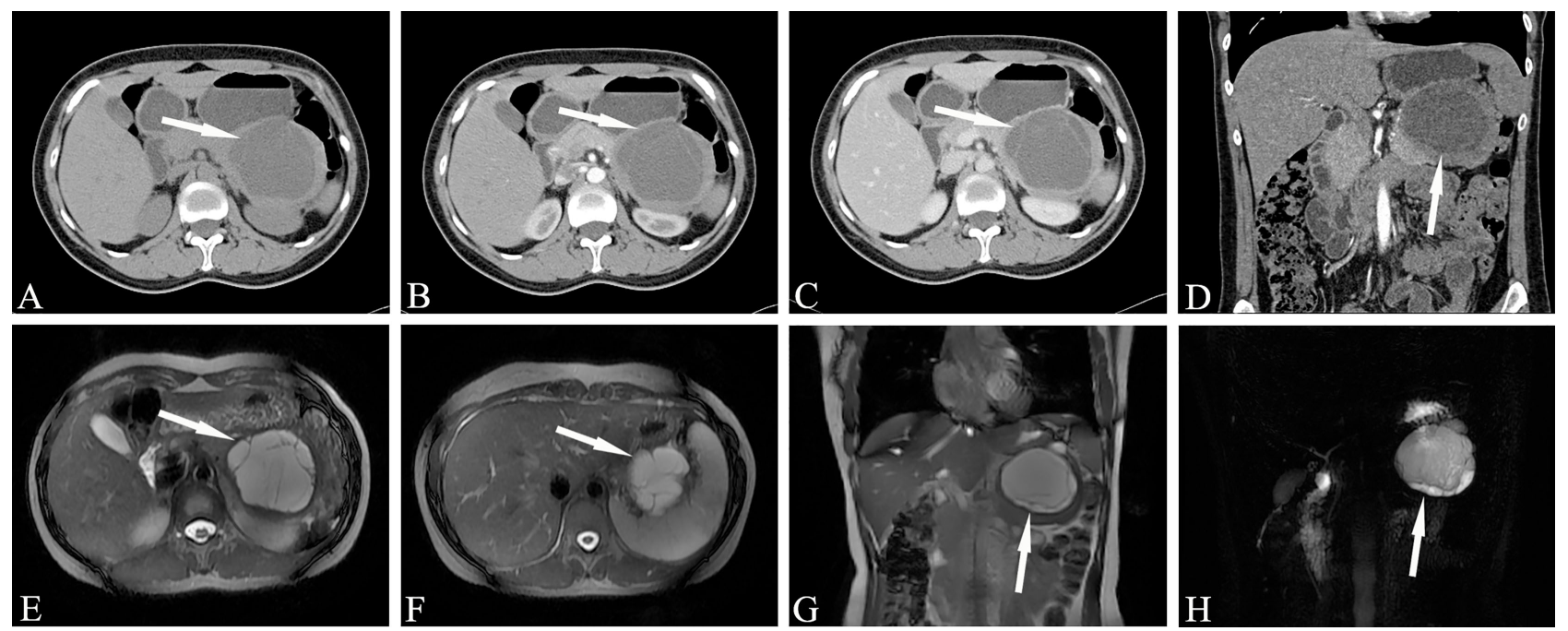

FIGURE 5 | Mucinous cystic neoplasms, MCN (Female, 33y, physical examination revealed a pancreatic mass for one month). The sequence distribution of images is the same as that in Figure 2. (A-D) Huge cystic mass in the body and tail of the pancreas, irregular in shape, high tension, watery low density, thin and uniform cyst wall, multiple thin septum and small walled cysts can be seen in the cyst, and the septation and mild cyst wall can be seen enhanced after contrast injection. (E-H) Magnetic resonance images showed clearer septum and small sacs. There were no signs of pancreatic duct dilatation. The white arrows in the Figures only indicate the location of the neoplasm.

benign and malignant pancreatic mucinous cystadenoma. Since the blood supply of the tumor is mainly concentrated in the cyst wall and septum, enhancement may appear after an enhanced scan.

\section{The CT and MR Manifestations of SCN}

CT scans of serous microcystic adenoma show a clear boundary, lobulation, and a mass formed by several small water-like cysts. The diameter of a single capsule is usually less than $2 \mathrm{~cm}$. Starshaped central scars can be seen in the lesions, and calcifications usually occur in the central scars. The enhanced scan shows progressive medium-strength enhancement of the central scars. It is often difficult to identify the tumor septum when it is thin, and enhancement can help with visualization when it is thicker. Star-shaped central scarring with or without calcification is considered to be a specific manifestation of serous microcystic adenoma. CT showing the intratumoral septum, central scar and size of the cyst is key to the imaging diagnosis of microserous microcystic adenoma. Sometimes serous microcystic adenomas are composed of numerous tiny vesicles, which show a honeycomb or spongy appearance, and most of the single cysts are one to several millimeters long (Figure 6). Clear edges and a cystic space with soft tissue structure can be seen on plain CT scan, and moderate enhancement is observed during the enhanced scan. At this time, it should be distinguished from SPN. When the lesion wall is thick, the internal components are complicated, and there is bleeding, calcification, liquefaction and necrosis, a solid pseudopapillary tumor is indicated.

MR shows serous cystadenoma basically similar to the CT appearance. However, the ability of MR to display calcification is weaker than that of CT, and MR can better display intracapsular hemorrhage, separation, and capsule wall. it can reveals more soft tissue information than CT scans. Soft tissue display can provide more information than CT. In addition, the display of the pancreatic duct and bile duct is more valuable for the differential diagnosis of diseases. Serous microcystic adenoma showed typical polycystic or honeycomb changes on MRI. The contents of the sac are a clear protein-containing liquid, generally showing a liquid signal shadow of long $\mathrm{T}_{1}$ and long $\mathrm{T}_{2}$. Sometimes the cyst cavity shows a slightly low signal shadow on $\mathrm{T}_{2} \mathrm{WI}$, which is caused by the local fluid concentration in the cyst cavity and the high protein content. Small cysts can only sometimes be a few millimeters. Intensified scanning of the cyst wall and separation are often mildly continuously enhanced. The lobulated contour, as if the collapsed wall dumped toward the center, is a feature of microcystic serous cystadenoma caused by the traction of the central star-shaped scar; the central scar sometimes has calcification, which appears to be sunlightradiating on $\mathrm{CT}$, with certain characteristics, but MRI has obvious shortcomings in showing scar tissue calcification.

MRI can almost show the cyst wall and the space within it, even for tumors with small diameters. The intratumoral septum can be seen more clearly on $\mathrm{T}_{2} \mathrm{WI}$. Serous tumor microcystic adenoma of the pancreas generally does not cause dilation of the CBD and PD. Although serous microcystic adenoma of the pancreatic head is adjacent to the $\mathrm{CBD}$, there are few signs of abnormal compression or obstruction in the bile duct system. This may be due to the tumor's soft body and slow growth, which does not compress the bile duct, also proving that the tumor is not aggressive. However, a small number of cases of serous microcystic adenoma with mild dilation of the pancreatic duct or CBD have also been reported. Mild pancreatic duct widening may be caused by mild compression changes or mild inflammatory changes in the pancreas. The 

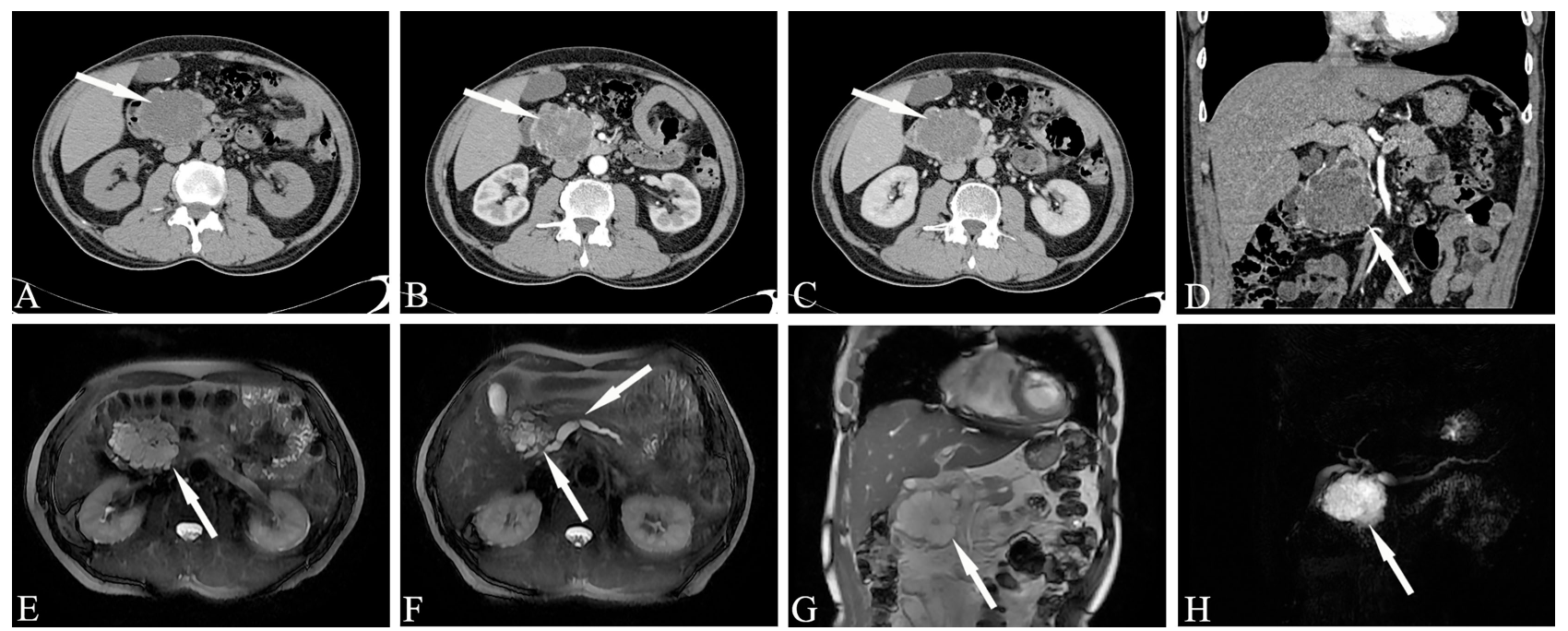

FIGURE 6 | Serous microcystic adenoma, SMA (Male, 65y, physical examination revealed a pancreatic mass for one month). The sequence distribution of images is the same as that in Figure 2. (A-D) Polycystic or honeycomb cystic foci in the head of the pancreas, with a lobulated outline, like a collapsed wall dumping to the center, and slightly continuous enhancement of the cyst wall and septum. Punctate calcification can be seen in the capsule wall. The enhanced scan shows progressive medium-strength enhancement of the central scars. (E-H) Magnetic resonance imaging reveals microcapsule-like structures more clearly and the MPD was slightly dilated. A stellate scar can be seen in the center of the lesion. The white arrows in the Figures only indicate the location of the neoplasm.

relationship between the cyst cavity and the pancreaticobiliary duct is of great significance to the diagnosis and differential diagnosis of this disease.

Serous microcystic adenomas caused by pancreatic tail duct dilatation should be differentiated from BD-IPMN. MRCP can easily determine the relationship between the neoplasm and the pancreatic duct. Microcystic cystadenoma with typical changes and other pancreatic cystic tumors are not difficult to distinguish. Serous oligocystic adenoma tumors show typical unicystic or multicystic changes on MRI. Cystic lesions are larger than serous microcystic adenomas. The lesions are clearly separated from the surrounding pancreas, and the edges are smooth. The characteristics of the signal of the cyst contents and the relationship with the pancreaticobiliary duct are roughly similar to those of serous microcystic adenoma. There is no sign of communication between the cyst cavity of the lesion and the pancreatic duct, and the adjacent CBD often shows no obvious compression or obstruction (Figure 7).

Solid serous cystadenoma contains a large number of fibrous interstitial blood vessels, and there is no cyst in the tumor according to general pathology. Only tiny cysts can be seen under the microscope, with abundant interstitial blood vessels (Figure 8). This type of cystadenoma is rare. Because the capsule is very small, it is difficult to display watery signals in the lesion on $\mathrm{T}_{2} \mathrm{WI}$, which often leads to the misdiagnosis of pancreatic neuroendocrine tumors.

\section{CT and MR Manifestations of SPN}

Regarding the typical CT appearance of a SPN, the solid part of the pancreas is slightly low-density, and cystic necrosis is shown as a lower-density area. The pathological basis that causes uneven density and mixed signals is tumor cystic transformation, hemorrhage, and the calcification of lesions(Figure 9). The distribution of cystic and solid components are also different; they can exist alternately, solid components can be located around the tumor, or multiple cysts of different sizes can be located at the edge of the tumor. Pathologically, the tumor cells in the pseudopapillary area form branched pseudopapillae with slender fibrous blood vessels as the axis. The cells are arranged in nests or lumps and in multiple layers. They are far away from the tumor cells around the blood vessels and are prone to degeneration. Necrosis, liquefaction and cystic changes can occur. Histologically, bleeding is prone to occur due to the large number of fragile, thin-walled blood vessels and the lack of a strong stent structure. Hemorrhage is one of the characteristics of this tumor.

Bleeding can occur in the cystic part or the solid part, showing gel-like or cystic tissue; the cystic and solid components of CT are scattered and patchy and show a high density. Calcification in the lesion is more common and can manifest in various ways: small spots, diffuse calcification, incomplete arc-shaped calcification of the envelope (Figure 9), and sometimes complete arc-shaped calcification. If the lesion is mainly cystic, most of the cyst is not strengthened, and a few solid parts inside are obviously strengthened, which are distributed in the low-density liquid tissue in the form of sheets, forming the so-called "floating cloud sign" (Figure 10). The surrounding envelope is obviously enhanced. In the case of a cystic solid structure, the solid part of the arterial phase is mostly papillary or wall nodular enhancement. For the solid structure, the solid part of the arterial phase is slightly enhanced, and the parenchymal and delayed phases are further strengthened, showing progressive filling, but both are lower than the degree of pancreatic parenchymal enhancement. 

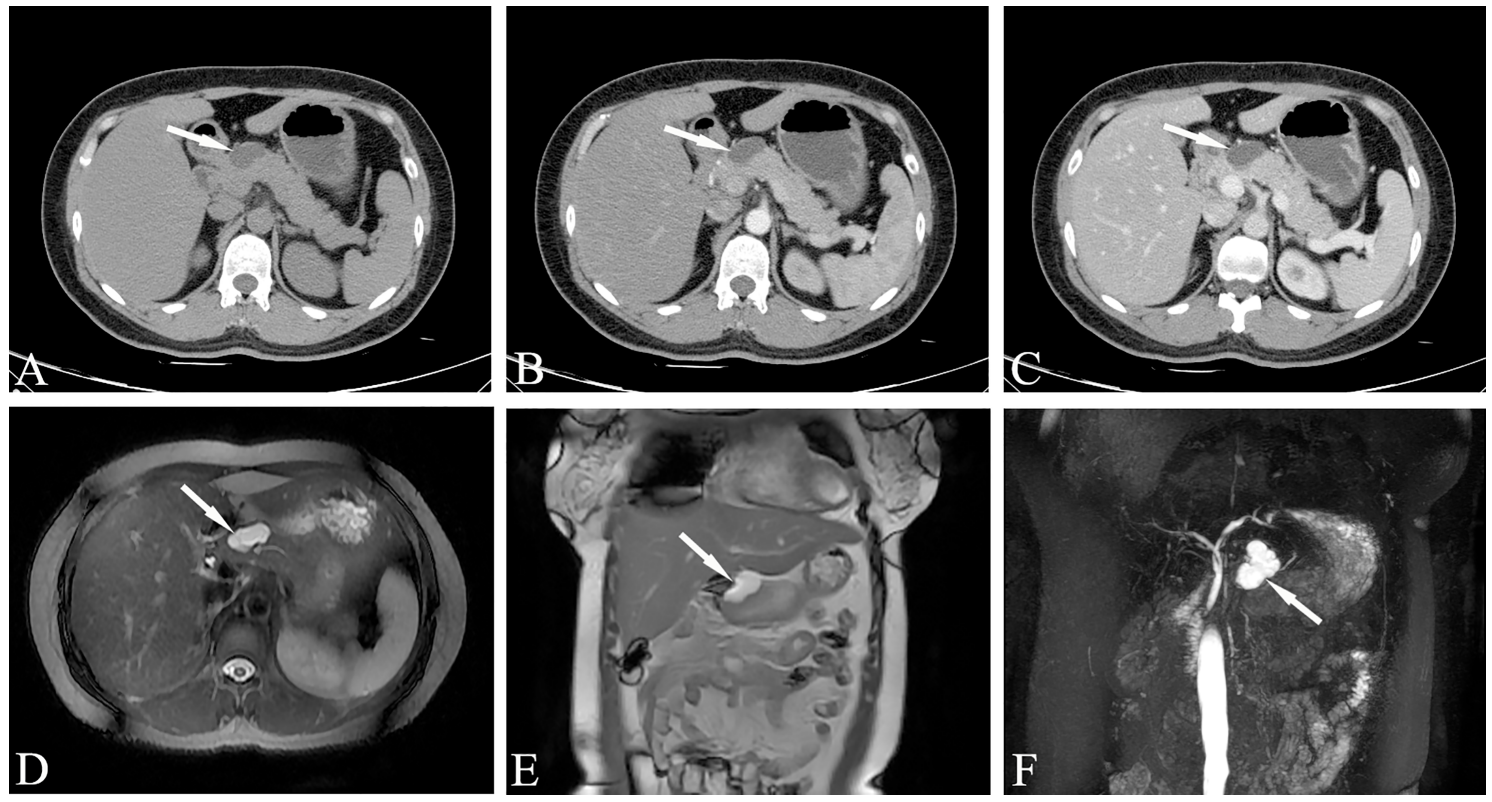

FIGURE 7 | Serous oligocystic adenoma, SOA (Female, 39y, physical examination revealed a pancreatic mass for two weeks). (A-C) The CT plain scan, arterial phase and venous phase at the same level; (D) $\mathrm{T}_{2}$-weighted cross-sectional image; (E). $\mathrm{T}_{2}$-weighted coronal image; (F) MRCP reconstruction map; (A-C) Lowdensity cystic mass in the neck of the pancreas with clear boundary and uniform density, no enhancement on dynamic contrast enhancement phase. (D-F) Magnetic resonance fat suppression $\mathrm{T}_{2} \mathrm{WI}$ showed a small cyst and a thin-walled separation next to the large cyst, and no signs of pancreatic duct dilatation. The white arrows in the Figures only indicate the location of the neoplasm.

For larger lesions, where cystic and solid lesions are often the main focus, the cystic component is not enhanced after enhancement, and the solid and cystic structures are clearly demarcated. Note that the pancreatic tissue has a "cup-mouth" boundary (Figure 10). Although the lesion is sometimes large, the MPD or CBD is generally not dilated. In a few cases, it may be slightly dilated, usually due to tumor compression of the adjacent duct. MRCP shows expansion of the MPD more intuitively than MDCT. A larger SPT can cause compression of adjacent blood vessels in the portal vein, splenic vein, inferior vena cava, and renal vein.

The MRI scan of SPN showed tumors with mixed signals on $\mathrm{T}_{1} \mathrm{WI}$ and $\mathrm{T}_{2} \mathrm{WI}$ and slightly hyper-intensity on DWI. The basis for the confounding of tumor signals is the tumor's cystic degeneration, necrosis, hemorrhage and calcification. MRI is more sensitive to the detection of tumor hemorrhage than CT. Usually, hemorrhage MRI shows a hyper-intensity on $\mathrm{T}_{1} \mathrm{WI}$ and a hyper- or hypo-intensity on $\mathrm{T}_{2} \mathrm{WI}$. Due to the coexistence of blood and other liquid components, signs of stratification can be seen, showing liquid levels. MRCP helps to show dilatation of the pancreaticobiliary duct.

\section{Endoscopic Ultrasound With Fine-Needle Aspiration}

Recently, EUS has been recognized as an essential diagnostic tool for PCN management. When the results of the radiological diagnosis of malignant tumors are certain and/or when the PCNs are considered to have clinical or radiological characteristics, EUS is nominated as a second-line examination method after CT/MRI. Because the stomach and pancreas are adjacent to each other in the body, the EUS transducer can be placed close to the pancreas, and the gland can be clearly imaged. In this way, the pancreatic cyst wall and its contents can be evaluated in detail, and internal septae and solid areas within the cysts can be differentiated.

One study (77) showed that EUS is the best diagnostic method for differentiating nonneoplastic cysts and PCNs and to characterize PCNs, being superior to both CT and MRI. However, another large multicenter study reported the opposite result: the accuracy of applying EUS to diagnose mucinous versus nonmucinous cysts was only $51 \%$ (16). It is speculated that one of the limitations of EUS may be due to different interpretations among endoscopists. Another study revealed that the accuracy of detecting neoplasms with malignant potential ranged from $40 \%$ to 93\% among 8 different endoscopists invited to interpret the same EUS procedure (78).

Making an accurate diagnosis with cross-sectional imaging and EUS alone is challenging, so EUS-FNA (fine needle aspiration) is frequently employed to obtain a cyst aspirate. Cyst fluid cytology suffers from poor sensitivity, which is specific. During EUS, various analyses (cytology, biochemistry and molecular) of pancreatic cyst fluid acquired from FNA can observably increase the accuracy of diagnosis $(1,16,79)$. In terms of the rate of correct diagnosis, EUS-FNA increased the accuracy by $36 \%$ after CT and the accuracy by $54 \%$ after MRI ( 80$)$. The risk of infection, hemorrhage, and pancreatitis of EUS-FNA 

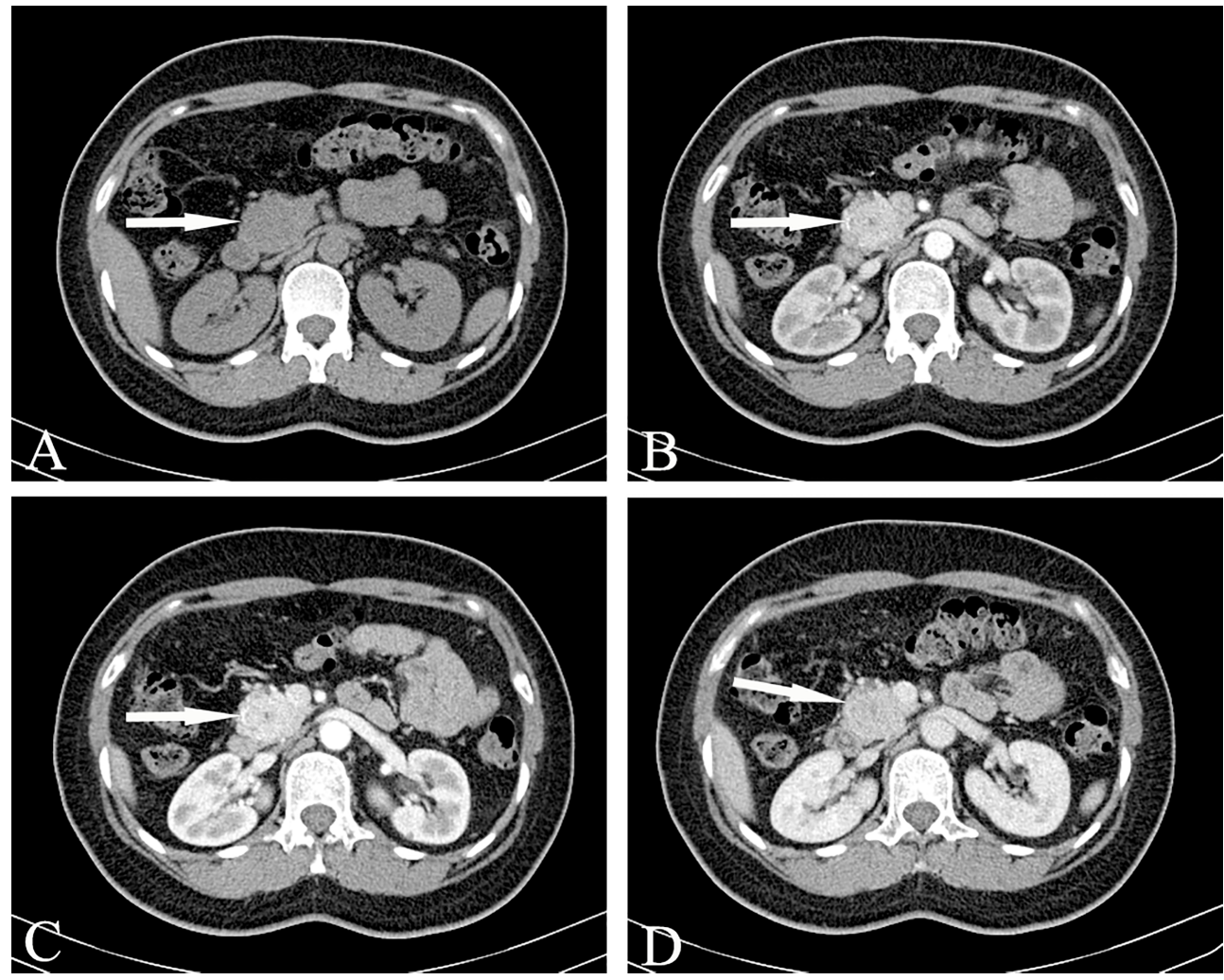

FIGURE 8 | Solid serous cystadenomas, SSCA (Female, 43y, physical examination revealed a pancreatic mass for three weeks). (A-D) The CT plain scan, arterial phase, portal and venous phase at the same level; (A) The CT plain scan showed a solid mass in the head of the pancreas, with a CT value of about $31 \mathrm{HU}$. (B) In the arterial phase, the mass of the pancreatic head was obviously enhanced, with a CT value of about 123HU. The focal low enhancement area can be seen in the center. (C) In the portal phase, the neoplasm showed progressive significantly enhancement, at this time, the CT value was 163HU; (D) The contrast wash-out can be seen in the venous phase of the mass, with a CT value of about $120 \mathrm{HU}$. The overall manifestation was solid tumor with rich blood supply of pancreas. The white arrows in the Figures only indicate the location of the neoplasm.

increases compared to noninvasive imaging, while most studies have shown that its benefits outweigh the risks $(81,82)$.

\section{Cytology}

EUS-FNA is a commonly used method of diagnosing IPMNs. However, the interpretation of cytological features relies on clinical and radiological findings. The presence of large amounts of thick mucin in the correct clinical setting can only suggest a diagnosis of IPMN. In contrast, it is difficult to distinguish the presence of limited mucin and low-grade mucinous epithelium from the presence of normal gastric epithelium (4). Compared with low-grade IPMN, the following features are more supportive of differentiating HGD IPMN: background of necrosis, aberrant chromatin patterns (hypochromasia or hyperchromasia), the presence of large vacuole single cells, significant nuclear irregularities, increased nuclearplasmic ratio, and small cell sizes $(\leq 12 \mu \mathrm{m}$ duodenal cell) (83). Furthermore, The high-grade atypia of IPMNs tend to be larger $(\geq 30$ $\mathrm{mm})$, have enhanced mural nodules $(\geq 5 \mathrm{~mm})$ (84) or have solid contents and dilated MPD ( $\geq 5 \mathrm{~mm})$ (85).

Differentiating between IPMN and MCN is also difficult for cytopathologists. Due to involvement with the pancreatic duct observed with IPMN but not MCN, correlation with radiologic findings can significantly facilitate diagnosis compared to others. At the same time, mucinous cystic tumors develop almost entirely in females, and presence of an ovarian stroma is pathognomonic. Aspirates of low-grade MCNs (mucinous cystic adenomas) that account for more than $75 \%$ of MCNs will show honeycomb sheets of bland mucin-containing epithelium but often lack the presence of complex papillary architecture compared to high-grade MCNs (4). The mucincontaining nucleus has a smooth contour, fine chromatin, and inconspicuous nucleoli (86).

EUS with FNA for SCNs has both low specificity and sensitivity, SCNs usually contain hemosiderin-laden macrophages and paucicellular cells with clear or hemorrhagic backgrounds. The highly vascularized fibrous septa of the SCN leads to the hemorrhagic nature of these specimens. SCNs do not involvement with the PD system and have low CEA levels, with cysts are often filled of clear-yellow serous fluid with low viscosity, compared with IPMNs. The cells of SCNs are bland. The nucleus is round, and the contour is smooth. Chromatin is evenly distributed in the nucleus, and the nucleoli are inconspicuous. When the background contains mucin, 

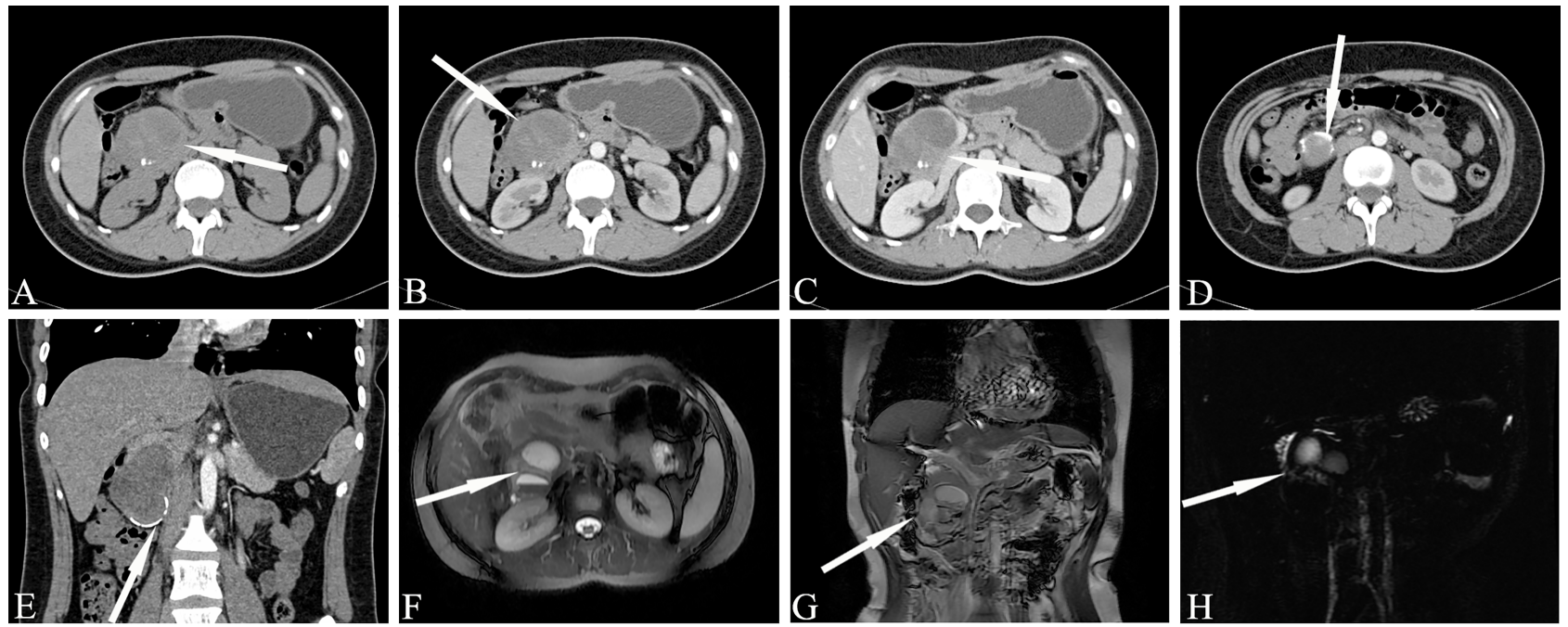

FIGURE 9 | Solid pseudopapillary neoplasm, SPN (Female, 28y, physical examination revealed a pancreatic mass for a week). (A-C) The CT plain scan, arterial phase and venous phase at the same level; (D) Coronal image in arterial phase. (E) Arterial phase cross-sectional image at another level of the same patient. (F). $T_{2}$-weighted cross-sectional image; (G) T2-weighted coronal image; (H). MRCP reconstruction map; (A) The CT plain scan showed a low-density mass in the head of the pancreas with cystic degeneration. Calcification was visible in the mass. (B-E) The solid component reinforcement was not obvious. The incomplete arc-shaped calcification of the envelope can be seen. (F-H) MRI shows old hemorrhagic signal with fluid-fluid level. There were no signs of pancreatic duct dilatation. The white arrows in the Figures only indicate the location of the neoplasm.
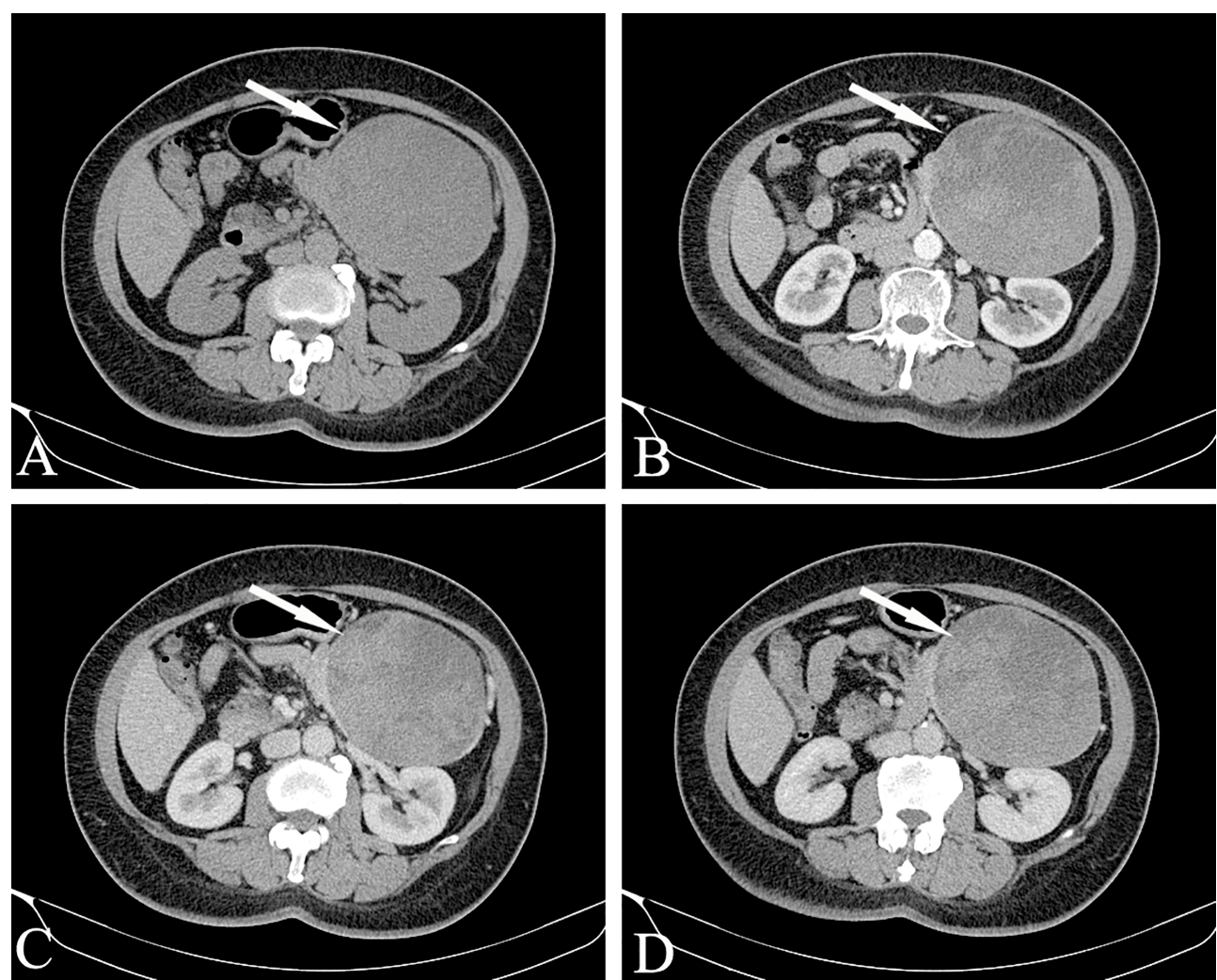

FIGURE 10 | Solid pseudopapillary neoplasm, SPN (Female, 58y, physical examination revealed a pancreatic mass for one month). (A-D) The CT plain scan, arterial phase, portal phase and venous phase at the same level. The lesions are large in size, with cystic and solid components visible inside. The cystic part is not enhanced, but the internal solid components are enhanced and distributed in patches in low-density liquid tissue, showing the "floating cloud sign". Besides, note that the pancreatic tissue had a "cup-mouth" boundary. The white arrows in the Figures only indicate the location of the neoplasm. 
associate with CEA levels and radiologic imaging would be cautiously exclude MCN. Macroscopically, SCNs are usually arranged around star-shaped scars, which show cysts with a distinctive spongy or honeycomb appearance.

The contents of hypercellular smears shown by EUS-guided FNA of SPTs include slender papillary fragments with fibrous vascular stalks and perivascular myxoid matrix. They are arranged by monomorphic cubic cells into cohesive groups and isolated cells. The neoplastic cells are round to oval, and the cytoplasmic boundary was unclear. The nucleus is grooved or bean-shaped, while the chromatin is fine-grained, and occasionally invisible or small nucleoli can be seen. Macroscopically, SPNs are large, with round to oval shapes, clear margin and fibrous pseudocapsules. SPNs are complex neoplasms with varies components (e.g., solid, cystic, hemorrhagic, and necrotic). Cystic degeneration is a common phenomenon that occurs during progression. Moreover, the larger the neoplasm is, the more obvious the cystic component $(45,87)$.

\section{Tumor Markers}

Studies have shown that pancreatic cyst fluid analysis of tumor markers and molecular markers can help characterize PCNs. At present, perfect biomarker testing for detecting pancreatic tumor has not yet been developed. The most commonly used blood test to monitor and detect pancreatic cancer is the serum marker CA 19-9. However, its sensitivity is limited, especially for small malignancies (88). When CEA levels > $192 \mathrm{ng} / \mathrm{mL}$, the sensitivity and specificity of the diagnosis of mucus lesions are $73 \%$ and $84 \%$, respectively (16). Negative and positive predictive values for mucin etiology are both $94 \%$ when CEA levels $<5 \mathrm{ng} /$ $\mathrm{mL}$ or $>800 \mathrm{ng} / \mathrm{mL}$ (17). The utilization of CEA using pancreatic cyst fluid to diagnose malignant cysts is less effective, as a previous meta-analysis suggested that both the diagnostic sensitivity and the specificity were 63\% (89). Chemical analysis of liquid CEA and amylase levels may be helpful, but this approach cannot differentiate between MCNs and IPMNs. Elevated CEA can be used as a marker to distinguish between mucinous and non-mucinous cysts rather than benign or malignant cysts. When the critical value of CEA was $\geq 192 \sim 200$ $\mathrm{ng} / \mathrm{mL}$, the accuracy increased to $80 \%$ for the diagnosis of mucinous cysts, showing high specificity but low sensitivity (16).

The current view is that serous cystic adenomas originate from centroacinar cells, where staining for cytokeratins and calretinin is positive but staining for CEA, mucin, estrogen receptors, and progesterone receptors is negative. SCN and ductal adenocarcinoma and neuroendocrine tumors can be distinguished by inhibin and calcarein, which were found to be helpful immunostaining markers in recent studies $(90,91)$.

\section{Molecular Markers}

To compensate for the limitations posed by cytology and tumor markers, specific molecular markers for diagnosing PCNs and predicting malignant tumors are currently being developed. A molecular DNA analysis method for pancreatic cyst fluid is currently on the market. However, a molecular analysis method for cyst fluid is still in development. KRAS mutations support the diagnosis of mucous cysts more accurately, but
KRAS does not always indicate a malignant cyst. It may be helpful to use GNAS mutations to differentiate between obvious mucinous cysts and indolent cysts that can be managed conservatively (92). Genetic analysis showed that the dual mutations in KRAS and GNAS were highly specific for IPMN. Compared with MCNs and SCN (lack GNAS codon 201 mutations), several research found that mutations in GNAS codon 201 are present in some IPMNs (41\%-66\%) and can even reach $74 \%$ to $100 \%$ in enteric IPMN (18-20).

With the aim of distinguishing MCNs from other PCNs, such as IPMN and SPN, some research have revealed that MCN is a kind of cystic neoplasm without the GNAS mutation and generally without the CTNNB1 mutation (21). KRAS mutations have been reported in MCNs (50\%-75\%) $(19,21)$. In serous cystadenomas, the absence of CTNNB1 mutation can be used to distinguish them from SPNs. In addition, KRAS and GNAS mutations are often expressed in IPMNs and MCNs rather than SCNs. There are a few studies of protein expression in SCAs. VEGF is a protein inhibited by a kind of tumor suppressor that is usually encoded by the VHL gene. In cysts or pancreatic duct fluid, VEGF-A levels can aid in diagnosis. The sensitivity and specificity for diagnosing SCAs were $100 \%$ and $97 \%$, respectively, when VEGF-A levels were $>8500 \mathrm{pg} /$ $\mathrm{mL}$ and $100 \%$ and $90 \%$, respectively, when VEGF-C levels were > $200 \mathrm{pg} / \mathrm{mL}$ (22). Combining both VEGF-A and VEGF-C provides $100 \%$ sensitivity and specificity for the diagnosis of SCA. In addition, VEGF-A (using a critical value of $>5000 \mathrm{pg} / \mathrm{mL}$ ) combined with CEA $(<10 \mathrm{ng} / \mathrm{mL})$ can detect SCAs with a sensitivity of $95.5 \%$ and a specificity of $100 \%$ (93).

Few research have focused on the glycoproteomics of SCAs. The research have shown that SCAs express MUC1 and MUC6 instead of MUC5AC, which provides proof that SCAs originate from pancreatic central cells and intralebar ducts $(23,24)$. An extracellular matrix protein implicated in pancreatic cancer called periostin was found to increase 8-fold in SCA cyst fluid compared to mucinous lesions (94). On the other side of the shield, serous cystadenomas are related to von Hippel Lindau (VHL) syndrome, while mutations in the VHL gene are present in all SCAs in patients with VHL syndrome. VHL loss-offunction mutations may also be reflected in the development of sporadic SCAs (95). The macrocyst (oligocyst) variant is a rare type of SCN with fewer but more numerous cysts and without a stellate central scar (96); solid variant, which is devoid of cysts; and mixed serous-neuroendocrine variant (91).

Studies have been performed to study several proteins related to the above genes in SPN tissue: B-catenin, androgen receptor, lymphoid enhancer-binding factor 1 (LEF1), and transcription factor for immunoglobulin heavy-chain enhancer 3 (TFE3) (25). Among them, B-catenin has a sensitivity of $98.9 \%$ and a specificity of $97 \%$ for the diagnosis of SPT, which is the most sensitive indicator of diagnosis. The combination of LEF1 and TFE3S increases the sensitivity to $100 \%$ but decreases the specificity to $91.9 \%$. Another investigation explored the use of B-catenin to diagnose SPT, reporting a $100 \%$ sensitivity and $87 \%$ specificity (26). The combined application of B-catenin, TEF3, and SOX11 can be used to distinguish SPN, with a sensitivity and specificity of $97 \%$. These tissue findings are also relevant for 
EUS-FNA biopsy samples (27). The absence of KRAS, GNAS, or RNF43 can distinguish SPTs from other PCNs. Because of the good prognosis of SPNs, complete resection of these inert neoplasms can be cured.

\section{THE MANAGEMENT OF PCNS}

Because of the significant overlap in the morphology of benign and premalignant lesions, characterizing and managing PCN poses a substantial dilemma for the clinical arena. However, compared with clinical and radiological suspicion, the patients are the most important parameter leading to clinical decisionmaking in surgery treatment. Patients fitness for surgery are continuous variable that should be considered in terms of age, life expectancy, health status, degree of frailty, patient preference, motivation for surgery, and availability of benefit. This parameter is crucial because the overall malignancy rate of PCNs is low. Each patient should be carefully evaluated by clinicians according to the patient's own situation after adequate consultation. Another significant factor in the final decision is the surgery type, as pancreaticoduodenectomy and distal pancreatectomy have different responsibilities in terms of morbidity, mortality, and sequelae.

There are two aspects that should guide the management of IPMN (1): whether the IPMN is malignant and (2) whether the IPMN will become malignant during a patient's lifetime. Clinicians still face the problem of detecting the presence of a malignancy in IPMN and determining its future malignant potential (97). According to the 2012 international consensus guidelines (44), surveillance of BD-type IPMN without "high-risk stigmata" was recommended based on the size stratification. On the basis of American Gastroenterological Association Institute guidelines, patients with pancreatic cysts $<3 \mathrm{~cm}$ without a solid component or a dilated pancreatic duct should undergo MRI at 1 year. If there is no change in size or characteristics, they should undergo MRI every 2 years afterward for a total of 5 years (70).

In the last 20 years, management recommendations for patients with IPMN have changed dramatically along with advances in our knowing of the natural history of this neoplasm. The reason for this evolution is that various studies have identified clinical, imaging and biologic predictors that may correctly distinguish IPMN with HGD and IC. Models with remarkable accuracy are being developed by combining clinical and imaging characteristics with promising cyst fluid markers. Given the relative rarity of this disease, enhancing constant international collaboration is necessary to successfully obtain a prevention strategy to reduce the incidence of pancreatic cancer arising from IPMN. Patients who have suspected findings but without absolute indications for surgery should undergo CEEUS. For patients in whom it has been difficult to confirm malignancy under endoscopy, further development of the disease should be closely monitored by MRI/MRCP, tumor markers, and CE-EUS. Once an SCN is detected, then the focus should be excision and long-term monitoring based on questions surrounding symptoms of local growth and progression, not cancer development.
If patients are doubted of having IPMNS, MCNs, and SPTs, appropriate lymphadenectomy and negative resection margins based on intraoperative frozen section assessment should be considered during surgical resection to completely remove the tumor. Given that the disease is usually malignant, parenchymasparing pancreatectomy is not a safe procedure for whole PCN cases. Overall, it should be considered only for selected cases or for SCNs.

However, it has not been demonstrated that prolonged followup reduces cancer-related mortality, but all these studies revealed that cyst stability over $5 y$ does not exclude the risk of future progression to pancreatic cancer, and thus, there is a lifelong risk of malignancy. Therefore, follow-up should be continued due to the importance of repeated observations for risk stratification. Currently, several follow-up schedules have been suggested in the current guidelines $(92,98)$; unfortunately, none of these schedules have been shown to be highly cost-effective. In general, the authors recommend that MRI/MRCP and oncological markers should be followed-up every 6 months for the 1st year in the absence of the suspicious features mentioned above. In the absence of progression, it is necessary to maintain follow-up with MRI/MRCP and serum markers for 12 or 18 months.

\section{CONCLUSIONS}

In recent years, the incidence of PCLs, especially PCNs, has increased daily. Due to the particularity of its anatomical location, the complexity of endocrine function, the diversity of pathological types, and the unsatisfactory prognosis, clinicians have become a great concern. The key issue is early diagnosis and early treatment, so the imaging diagnosis of pancreatic cystic tumors shows important value in diagnosis. The accuracy of preoperative imaging diagnosis is essential to improve clinicians' confidence in surgery and individualized management. In conclusion, we hope that in the future, imaging biomarkers can be used along with histopathology to provide greater theoretical support for the precise treatment of tumor patients.

\section{AUTHOR CONTRIBUTIONS}

$\mathrm{FH}$ and $\mathrm{YH}$ are the co-first authors of this paper and have contributed equally to this work. WP and TT are the cocorresponding authors. They are responsible for the quality of the review. All authors made substantial contributions to all of the following: (1) the conception and design of the study, or acquisition of data, or analysis and interpretation of data, (2) drafting the article or revising it critically for important intellectual content, (3) final approval of the version to be submitted. Publication is approved by all authors. There are no conflicts of interests associated with this work.

\section{FUNDING}

The research was supported by the National Natural Science Foundation of China (No. 81971687/No. 81801702) 


\section{REFERENCES}

1. van Huijgevoort N, Del CM, Wolfgang CL, van Hooft JE, Besselink MG. Diagnosis and Management of Pancreatic Cystic Neoplasms: Current Evidence and Guidelines. Nat Rev Gastroenterol Hepatol (2019) 16:676-89. doi: 10.1038/s41575-019-0195-x

2. Mamone G, Barresi L, Tropea A, Di Piazza A, Miraglia R. MRI of Mucinous Pancreatic Cystic Lesions: A New Updated Morphological Approach for The Differential Diagnosis. Updates Surg (2020) 72:617-37. doi: 10.1007/s13304020-00800-y

3. Perri G, Marchegiani G, Frigerio I, Dervenis CG, Conlon KC, Bassi C, et al. Management of Pancreatic Cystic Lesions. Dig Surg (2020) 37:1-9. doi: $10.1159 / 000496509$

4. Abdelkader A, Hunt B, Hartley CP, Panarelli NC, Giorgadze T. Cystic Lesions of the Pancreas: Differential Diagnosis and Cytologic-Histologic Correlation. Arch Pathol Lab Med (2020) 144:47-61. doi: 10.5858/arpa.2019-0308-RA

5. Dalal V, Carmicheal J, Dhaliwal A, Jain M, Kaur S, Batra SK. Radiomics in Stratification of Pancreatic Cystic Lesions: Machine Learning in Action. Cancer Lett (2020) 469:228-37. doi: 10.1016/j.canlet.2019.10.023

6. Keegan MJ, Paranandi B. Diagnosis and Management of Pancreatic Cystic Neoplasms. Frontline Gastroenterol (2019) 10:300-8. doi: 10.1136/flgastro2018-101122

7. Yoon WJ, Brugge WR. Pancreatic Cystic Neoplasms: Diagnosis and Management. Gastroenterol Clin North Am (2012) 41:103-18. doi: 10.1016/ j.gtc.2011.12.016

8. Brugge WR, Lauwers GY, Sahani D, Fernandez-del CC, Warshaw AL. Cystic Neoplasms of the Pancreas. N Engl J Med (2004) 351:1218-26. doi: 10.1056/ NEJMra031623

9. Kearns M, Ahmad NA. Diagnosis and Management of Pancreatic Cystic Neoplasms. Curr Treat Options Gastroenterol (2017) 15:587-602. doi: 10.1007/s11938-017-0162-y

10. Pezzilli R, Buscarini E, Pollini T, Bonamini D, Marchegiani G, Crippa S, et al. Epidemiology, Clinical Features and Diagnostic Work-Up of Cystic Neoplasms of the Pancreas: Interim Analysis of the Prospective PANCY Survey. Dig Liver Dis (2020) 52:547-54. doi: 10.1016/j.dld.2020.02.003

11. Distler M, Kersting S, Niedergethmann M, Aust DE, Franz M, Rückert F, et al. Pathohistological Subtype Predicts Survival in Patients With Intraductal Papillary Mucinous Neoplasm (IPMN) of the Pancreas. Ann Surg (2013) 258:324-30. doi: 10.1097/SLA.0b013e318287ab73

12. Testini M, Gurrado A, Lissidini G, Venezia P, Greco L, Piccinni G. Management of Mucinous Cystic Neoplasms of the Pancreas. World J Gastroenterol (2010) 16:5682-92. doi: 10.3748/wjg.v16.i45.5682

13. Sakorafas GH, Smyrniotis V, Reid-Lombardo KM, Sarr MG. Primary Pancreatic Cystic Neoplasms Revisited: Part II. Mucinous Cystic Neoplasms. Surg Oncol (2011) 20:e93-101. doi: 10.1016/j.suronc.2010.12.003

14. Wang GX, Wang ZP, Chen HL, Zhang D, Wen L. Discrimination of Serous Cystadenoma From Mucinous Cystic Neoplasm and Branch Duct Intraductal Papillary Mucinous Neoplasm in the Pancreas With CT. Abdom Radiol (NY) (2020) 45:2772-8. doi: 10.1007/s00261-020-02664-7

15. Chandwani R, Allen PJ. Cystic Neoplasms of the Pancreas. Annu Rev Med (2016) 67:45-57. doi: 10.1146/annurev-med-051914-022011

16. Brugge WR, Lewandrowski K, Lee-Lewandrowski E, Centeno BA, Szydlo T, Regan S, et al. Diagnosis of Pancreatic Cystic Neoplasms: A Report of the Cooperative Pancreatic Cyst Study. Gastroenterology (2004) 126:1330-6. doi: 10.1053/j.gastro.2004.02.013

17. van der Waaij LA, van Dullemen HM, Porte RJ. Cyst Fluid Analysis in the Differential Diagnosis of Pancreatic Cystic Lesions: A Pooled Analysis. Gastrointest Endosc (2005) 62:383-9. doi: 10.1016/s0016-5107(05)01581-6

18. Lee LS, Doyle LA, Houghton J, Sah S, Bellizzi AM, Szafranska-Schwarzbach AE, et al. Differential Expression of GNAS and KRAS Mutations in Pancreatic Cysts. JOP (2014) 15:581-6. doi: 10.6092/1590-8577/2432

19. Wu J, Matthaei H, Maitra A, Dal Molin M, Wood LD, Eshleman JR, et al. Recurrent GNAS Mutations Define an Unexpected Pathway for Pancreatic Cyst Development. Sci Transl Med (2011) 3:66r-92r. doi: 10.1126/ scitranslmed.3002543

20. Furukawa T, Kuboki Y, Tanji E, Yoshida S, Hatori T, Yamamoto M, et al. Whole-Exome Sequencing Uncovers Frequent GNAS Mutations in
Intraductal Papillary Mucinous Neoplasms of the Pancreas. Sci Rep (2011) 1:161. doi: $10.1038 /$ srep00161

21. Springer S, Wang Y, Dal Molin M, Masica DL, Jiao Y, Kinde I, et al. A Combination of Molecular Markers and Clinical Features Improve the Classification of Pancreatic Cysts. Gastroenterology (2015) 149:1501-10. doi: 10.1053/j.gastro.2015.07.041

22. Yip-Schneider MT, Wu H, Dumas RP, Hancock BA, Agaram N, Radovich M, et al. Vascular Endothelial Growth Factor, a Novel and Highly Accurate Pancreatic Fluid Biomarker for Serous Pancreatic Cysts. J Am Coll Surg (2014) 218:608-17. doi: 10.1016/j.jamcollsurg.2013.12.019

23. Kosmahl M, Wagner J, Peters K, Sipos B, Klöppel G. Serous Cystic Neoplasms of the Pancreas: An Immunohistochemical Analysis Revealing Alpha-Inhibin, Neuron-Specific Enolase, and MUC6 as New Markers. Am J Surg Pathol (2004) 28:339-46. doi: 10.1097/00000478-200403000-00006

24. Reid MD, Choi H, Balci S, Akkas G, Adsay V. Serous Cystic Neoplasms of the Pancreas: Clinicopathologic and Molecular Characteristics. Semin Diagn Pathol (2014) 31:475-83. doi: 10.1053/j.semdp.2014.08.009

25. Kim EK, Jang M, Park M, Kim H. LEF1, TFE3, and AR are Putative Diagnostic Markers of Solid Pseudopapillary Neoplasms. Oncotarget (2017) 8:93404-13. doi: 10.18632/oncotarget.21854

26. Harrison G, Hemmerich A, Guy C, Perkinson K, Fleming D, McCall S, et al. Overexpression of SOX11 and TFE3 in Solid-Pseudopapillary Neoplasms of the Pancreas. Am J Clin Pathol (2017) 149:67-75. doi: 10.1093/ajcp/aqx142

27. Foo WC, Harrison G, Zhang X. Immunocytochemistry for SOX-11 and TFE3 as Diagnostic Markers for Solid Pseudopapillary Neoplasms of the Pancreas in FNA Biopsies. Cancer Cytopathol (2017) 125:831-7. doi: 10.1002/cncy.21931

28. Castellano-Megías VM, Andrés CI, López-Alonso G, Colina-Ruizdelgado F. Pathological Features and Diagnosis of Intraductal Papillary Mucinous Neoplasm of The Pancreas. World J Gastrointest Oncol (2014) 6:311-24. doi: 10.4251/wjgo.v6.i9.311

29. Mino-Kenudson M, Fernández-del CC, Baba Y, Valsangkar NP, Liss AS, Hsu M, et al. Prognosis of Invasive Intraductal Papillary Mucinous Neoplasm Depends on Histological and Precursor Epithelial Subtypes. Gut (2011) 60:1712-20. doi: 10.1136/gut.2010.232272

30. Salvia R, Crippa S, Falconi M, Bassi C, Guarise A, Scarpa A, et al. Branch-Duct Intraductal Papillary Mucinous Neoplasms of the Pancreas: To Operate or Not to Operate? Gut (2007) 56:1086-90. doi: 10.1136/gut.2006.100628

31. Maguchi H, Tanno S, Mizuno N, Hanada K, Kobayashi G, Hatori T, et al. Natural History of Branch Duct Intraductal Papillary Mucinous Neoplasms of the Pancreas: A Multicenter Study in Japan. Pancreas (2011) 40:364-70. doi: 10.1097/MPA.0b013e31820a5975

32. Rodriguez JR, Salvia R, Crippa S, Warshaw AL, Bassi C, Falconi M, et al. Branch-Duct Intraductal Papillary Mucinous Neoplasms: Observations in 145 Patients Who Underwent Resection. Gastroenterology (2007) 133:72-9, 30910. doi: 10.1053 /j.gastro.2007.05.010

33. Yamada S, Fujii T, Murotani K, Kanda M, Sugimoto H, Nakayama G, et al. Comparison of the International Consensus Guidelines for Predicting Malignancy in Intraductal Papillary Mucinous Neoplasms. Surgery (2016) 159:878-84. doi: 10.1016/j.surg.2015.08.042

34. Maire F, Hammel P, Terris B, Paye F, Scoazec JY, Cellier C, et al. Prognosis of Malignant Intraductal Papillary Mucinous Tumours of the Pancreas After Surgical Resection. Comparison With Pancreatic Ductal Adenocarcinoma. Gut (2002) 51:717-22. doi: 10.1136/gut.51.5.717

35. Heckler M, Brieger L, Heger U, Pausch T, Tjaden C, Kaiser J, et al. Predictive Performance of Factors Associated With Malignancy in Intraductal Papillary Mucinous Neoplasia of the Pancreas. BJS Open (2018) 2:13-24. doi: 10.1002/ bjs5.38

36. Konstantinou F, Syrigos KN, Saif MW. Intraductal Papillary Mucinous Neoplasms of the Pancreas (IPMNs): Epidemiology, Diagnosis and Future Aspects. JOP (2013) 14:141-4. doi: 10.6092/1590-8577/1467

37. Bojanapu S, Kasi A. Pancreatic Mucinous Cystadenoma. (2022).

38. Yeh MM, Tang LH, Wang S, Robert ME, Zheng W, Jain D. Inhibin Expression in Ovarian-Type Stroma in Mucinous Cystic Neoplasms of the Pancreas. Appl Immunohistochem Mol Morphol (2004) 12:148-52. doi: 10.1097/00129039200406000-00009

39. Lennon AM, Wolfgang C. Cystic Neoplasms of the Pancreas. J Gastrointest Surg (2013) 17:645-53. doi: 10.1007/s11605-012-2072-6 
40. Levy MJ. Pancreatic Cysts. Gastrointest Endosc (2009) 69:S110-6. doi: 10.1016/j.gie.2008.12.011

41. Gress F, Gottlieb K, Cummings O, Sherman S, Lehman G. Endoscopic Ultrasound Characteristics of Mucinous Cystic Neoplasms of the Pancreas. Am J Gastroenterol (2000) 95:961-5. doi: 10.1111/j.1572-0241.2000.01976.x

42. Garces-Descovich A, Beker K, Castillo-Angeles M, Brook A, Resnick E, Shinagare S, et al. Mucinous Cystic Neoplasms of the Pancreas: HighResolution Cross-Sectional Imaging Features With Clinico-Pathologic Correlation. Abdom Radiol (NY) (2018) 43:1413-22. doi: 10.1007/s00261017-1326-x

43. Bai XL, Zhang Q, Masood N, Masood W, Zhang Y, Liang TB. Pancreatic Cystic Neoplasms: A Review of Preoperative Diagnosis and Management. J Zhejiang Univ Sci B (2013) 14:185-94. doi: 10.1631/jzus.B1200283

44. Tanaka M, Fernández-del CC, Adsay V, Chari S, Falconi M, Jang JY, et al. International Consensus Guidelines 2012 for the Management of IPMN and MCN of the Pancreas. Pancreatology (2012) 12:183-97. doi: 10.1016/ j.pan.2012.04.004

45. Papavramidis T, Papavramidis S. Solid Pseudopapillary Tumors of the Pancreas: Review of 718 Patients Reported in English Literature. J Am Coll Surg (2005) 200:965-72. doi: 10.1016/j.jamcollsurg.2005.02.011

46. Ren Z, Zhang P, Zhang X, Liu B. Solid Pseudopapillary Neoplasms of the Pancreas: Clinicopathologic Features and Surgical Treatment of 19 Cases. Int $J$ Clin Exp Pathol (2014) 7:6889-97.

47. Law JK, Ahmed A, Singh VK, Akshintala VS, Olson MT, Raman SP, et al. A Systematic Review of Solid-Pseudopapillary Neoplasms: Are These Rare Lesions? Pancreas (2014) 43:331-7. doi: 10.1097/MPA.0000000000000061

48. Yao J, Song H. A Review of Clinicopathological Characteristics and Treatment of Solid Pseudopapillary Tumor of the Pancreas With 2450 Cases in Chinese Population. BioMed Res Int (2020) 2020:2829647. doi: 10.1155/2020/2829647

49. Jutric Z, Rozenfeld Y, Grendar J, Hammill CW, Cassera MA, Newell PH, et al. Analysis of 340 Patients With Solid Pseudopapillary Tumors of the Pancreas: A Closer Look at Patients With Metastatic Disease. Ann Surg Oncol (2017) 24:2015-22. doi: 10.1245/s10434-017-5772-z

50. Reddy S, Cameron JL, Scudiere J, Hruban RH, Fishman EK, Ahuja N, et al. Surgical Management of Solid-Pseudopapillary Neoplasms of the Pancreas (Franz or Hamoudi Tumors): A Large Single-Institutional Series. J Am Coll Surg (2009) 208:950-7, 957-9. doi: 10.1016/j.jamcollsurg.2009.01.044

51. Dababneh Y, Mousa OY. Pancreatic Serous Cystadenoma. (2022).

52. George DH, Murphy F, Michalski R, Ulmer BG. Serous Cystadenocarcinoma of the Pancreas: A New Entity? Am J Surg Pathol (1989) 13:61-6. doi: 10.1097/ 00000478-198901000-00009

53. Jais B, Rebours V, Malleo G, Salvia R, Fontana M, Maggino L, et al. Serous Cystic Neoplasm of the Pancreas: A Multinational Study of 2622 Patients Under The Auspices of the International Association of Pancreatology and European Pancreatic Club (European Study Group on Cystic Tumors of the Pancreas). Gut (2016) 65:305-12. doi: 10.1136/gutjnl-2015-309638

54. Chu LC, Singhi AD, Haroun RR, Hruban RH, Fishman EK. The Many Faces of Pancreatic Serous Cystadenoma: Radiologic and Pathologic Correlation. Diagn Interv Imaging (2017) 98:191-202. doi: 10.1016/j.diii.2016.08.005

55. Jederán É. Importance of Conventional Pancreas Imaging: Ultrasound, CT, and MRI. Facts and Possibilities. Magy Onkol (2021) 65:237-49.

56. Harrington KA, Shukla-Dave A, Paudyal R, Do R. MRI of the Pancreas. J Magn Reson Imaging (2021) 53:347-59. doi: 10.1002/jmri.27148

57. Wang G, Dang H, Yu P, Liu H, Wu Y, Yao S, et al. Multiparameter Analysis Using (18)F-FDG PET/CT in the Differential Diagnosis of Pancreatic Cystic Neoplasms. Contrast Media Mol Imaging (2021) 2021:6658644. doi: 10.1155/ 2021/6658644

58. Chen S, Ren S, Guo K, Daniels MJ, Wang Z, Chen R. Preoperative Differentiation of Serous Cystic Neoplasms From Mucin-Producing Pancreatic Cystic Neoplasms Using a CT-Based Radiomics Nomogram. Abdom Radiol (NY) (2021) 46:2637-46. doi: 10.1007/s00261-021-02954-8

59. Wei R, Lin K, Yan W, Guo Y, Wang Y, Li J, et al. Computer-Aided Diagnosis of Pancreas Serous Cystic Neoplasms: A Radiomics Method on Preoperative MDCT Images. Technol Cancer Res Treat (2019) 18:1077091987. doi: 10.1177/ 1533033818824339

60. Sahani DV, Sainani NI, Blake MA, Crippa S, Mino-Kenudson M, Del-Castillo CF. Prospective Evaluation of Reader Performance on MDCT in Characterization of Cystic Pancreatic Lesions and Prediction of Cyst
Biologic Aggressiveness. AJR Am J Roentgenol (2011) 197:W53-61. doi: 10.2214/AJR.10.5866

61. Dmitriev K, Kaufman AE, Javed AA, Hruban RH, Fishman EK, Lennon AM, et al. Classification of Pancreatic Cysts in Computed Tomography Images Using a Random Forest and Convolutional Neural Network Ensemble. Med Image Comput Comput Assist Interv (2017) 10435:150-8. doi: 10.1007/978-3319-66179-7_18

62. Laffan TA, Horton KM, Klein AP, Berlanstein B, Siegelman SS, Kawamoto S, et al. Prevalence of Unsuspected Pancreatic Cysts on MDCT. AJR Am J Roentgenol (2008) 191:802-7. doi: 10.2214/AJR.07.3340

63. Sahani DV, Kambadakone A, Macari M, Takahashi N, Chari S, Fernandez-del CC. Diagnosis and Management of Cystic Pancreatic Lesions. AJR Am J Roentgenol (2013) 200:343-54. doi: 10.2214/AJR.12.8862

64. Sahani DV, Kadavigere R, Saokar A, Fernandez-del CC, Brugge WR, Hahn PF. Cystic Pancreatic Lesions: A Simple Imaging-Based Classification System for Guiding Management. Radiographics (2005) 25:1471-84. doi: 10.1148/ rg. 256045161

65. Lin XZ, Wu ZY, Li WX, Zhang J, Xu XQ, Chen KM, et al. Differential Diagnosis of Pancreatic Serous Oligocystic Adenoma and Mucinous Cystic Neoplasm With Spectral CT Imaging: Initial Results. Clin Radiol (2014) 69:1004-10. doi: 10.1016/j.crad.2014.05.003

66. Hanania AN, Bantis LE, Feng Z, Wang H, Tamm EP, Katz MH, et al. Quantitative Imaging to Evaluate Malignant Potential of IPMNs. Oncotarget (2016) 7:85776-84. doi: 10.18632/oncotarget.11769

67. Permuth JB, Choi J, Balarunathan Y, Kim J, Chen DT, Chen L, et al. Combining Radiomic Features With a miRNA Classifier may Improve Prediction of Malignant Pathology for Pancreatic Intraductal Papillary Mucinous Neoplasms. Oncotarget (2016) 7:85785-97. doi: 10.18632/ oncotarget.11768

68. Yang J, Guo X, Ou X, Zhang W, Ma X. Discrimination of Pancreatic Serous Cystadenomas From Mucinous Cystadenomas With CT Textural Features: Based on Machine Learning. Front Oncol (2019) 9:494:494. doi: 10.3389/ fonc.2019.00494

69. Curry CA, Eng J, Horton KM, Urban B, Siegelman S, Kuszyk BS, et al. CT of Primary Cystic Pancreatic Neoplasms: Can CT be Used for Patient Triage and Treatment? AJR Am J Roentgenol (2000) 175:99-103. doi: 10.2214/ ajr.175.1.1750099

70. Vege SS, Ziring B, Jain R, Moayyedi P. American Gastroenterological Association Institute Guideline on the Diagnosis and Management of Asymptomatic Neoplastic Pancreatic Cysts. Gastroenterology (2015) 148:819-22, e12-3. doi: 10.1053/j.gastro.2015.01.015

71. Lee KS, Sekhar A, Rofsky NM, Pedrosa I. Prevalence of Incidental Pancreatic Cysts in the Adult Population on MR Imaging. Am J Gastroenterol (2010) 105:2079-84. doi: 10.1038/ajg.2010.122

72. de Jong K, Bruno MJ, Fockens P. Epidemiology, Diagnosis, and Management of Cystic Lesions of the Pancreas. Gastroenterol Res Pract (2012) 2012:147465. doi: $10.1155 / 2012 / 147465$

73. Sandrasegaran K, Lin C, Akisik FM, Tann M. State-Of-the-Art Pancreatic MRI. AJR Am J Roentgenol (2010) 195:42-53. doi: 10.2214/ajr.10.4421

74. Lee HJ, Kim MJ, Choi JY, Hong HS, Kim KA. Relative Accuracy of CT and MRI in the Differentiation of Benign From Malignant Pancreatic Cystic Lesions. Clin Radiol (2011) 66:315-21. doi: 10.1016/j.crad.2010.06.019

75. Zhu S, Wang WT, Shang XS, Ni T, Wu WC, Lou WH, et al. Difference Analysis in Prevalence of Incidental Pancreatic Cystic Lesions Between Computed Tomography and Magnetic Resonance Imaging. BMC Med Imaging (2019) 19:43. doi: 10.1186/s12880-019-0341-5

76. Hoffman DH, Ream JM, Hajdu CH, Rosenkrantz AB. Utility of Whole-Lesion ADC Histogram Metrics for Assessing the Malignant Potential Of Pancreatic Intraductal Papillary Mucinous Neoplasms (IPMNs). Abdom Radiol (NY) (2017) 42:1222-8. doi: 10.1007/s00261-016-1001-7

77. Du C, Chai NL, Linghu EQ, Li HK, Sun LH, Jiang L, et al. Comparison of Endoscopic Ultrasound, Computed Tomography and Magnetic Resonance Imaging in Assessment of Detailed Structures of Pancreatic Cystic Neoplasms. World J Gastroenterol (2017) 23:3184-92. doi: 10.3748/wjg.v23.i17.3184

78. Ahmad NA, Kochman ML, Brensinger C, Brugge WR, Faigel DO, Gress FG, et al. Interobserver Agreement Among Endosonographers for the Diagnosis of Neoplastic Versus Non-Neoplastic Pancreatic Cystic Lesions. Gastrointest Endosc (2003) 58:59-64. doi: 10.1067/mge.2003.298 
79. Khalid A, Brugge W. ACG Practice Guidelines for the Diagnosis and Management of Neoplastic Pancreatic Cysts. Am J Gastroenterol (2007) 102:2339-49. doi: 10.1111/j.1572-0241.2007.01516.x

80. Khashab MA, Kim K, Lennon AM, Shin EJ, Tignor AS, Amateau SK, et al. Should We do EUS/FNA on Patients With Pancreatic Cysts? The Incremental Diagnostic Yield of EUS Over CT/MRI for Prediction of Cystic Neoplasms. Pancreas (2013) 42:717-21. doi: 10.1097/MPA.0b013e3182883a91

81. Lim SJ, Alasadi R, Wayne JD, Rao S, Rademaker A, Bell R, et al. Preoperative Evaluation of Pancreatic Cystic Lesions: Cost-Benefit Analysis and Proposed Management Algorithm. Surgery (2005) 138:672-9, 679-80. doi: 10.1016/ j.surg.2005.07.033

82. Wang KX, Ben QW, Jin ZD, Du YQ, Zou DW, Liao Z, et al. Assessment of Morbidity and Mortality Associated With EUS-Guided FNA: A Systematic Review. Gastrointest Endosc (2011) 73:283-90. doi: 10.1016/j.gie.2010.10.045

83. Pitman MB, Centeno BA, Daglilar ES, Brugge WR, Mino-Kenudson M. Cytological Criteria of High-Grade Epithelial Atypia in the Cyst Fluid of Pancreatic Intraductal Papillary Mucinous Neoplasms. Cancer Cytopathol (2014) 122:40-7. doi: 10.1002/cncy.21344

84. Anand N, Sampath K, Wu BU. Cyst Features and Risk of Malignancy in Intraductal Papillary Mucinous Neoplasms of The Pancreas: A Meta-Analysis. Clin Gastroenterol Hepatol (2013) 11:913-21.e59-60. doi: 10.1016/j.cgh. 2013.02.010

85. Salvia R, Fernández-del CC, Bassi C, Thayer SP, Falconi M, Mantovani W, et al. Main-Duct Intraductal Papillary Mucinous Neoplasms of the Pancreas: Clinical Predictors of Malignancy and Long-Term Survival Following Resection. Ann Surg (2004) 239:678-85, 685-7. doi: 10.1097/ 01.sla.0000124386.54496.15

86. Recine M, Kaw M, Evans DB, Krishnamurthy S. Fine-Needle Aspiration Cytology of Mucinous Tumors of the Pancreas. Cancer-Am Cancer Soc (2004) 102:92-9. doi: 10.1002/cncr.20052

87. Santini D, Poli F, Lega S. Solid-Papillary Tumors of the Pancreas: Histopathology. JOP (2006) 7:131-6.

88. Goggins M. Molecular Markers of Early Pancreatic Cancer. J Clin Oncol (2005) 23:4524-31. doi: 10.1200/JCO.2005.19.711

89. Ngamruengphong S, Bartel MJ, Raimondo M. Cyst Carcinoembryonic Antigen in Differentiating Pancreatic Cysts: A Meta-Analysis. Dig Liver Dis (2013) 45:920-6. doi: 10.1016/j.dld.2013.05.002

90. Marsh WL, Colonna J, Yearsley M, Bloomston M, Frankel WL. Calponin is Expressed in Serous Cystadenomas of the Pancreas But Not in Adenocarcinomas or Endocrine Tumors. Appl Immunohistochem Mol Morphol (2009) 17:216-9. doi: 10.1097/pai.0b013e31818claa6

91. Charville GW, Kao CS. Serous Neoplasms of the Pancreas: A Comprehensive Review. Arch Pathol Lab Med (2018) 142:1134-40. doi: 10.5858/arpa.20170195-RS

92. Tanaka M, Fernández-Del CC, Kamisawa T, Jang JY, Levy P, Ohtsuka T, et al. Revisions of International Consensus Fukuoka Guidelines for the
Management of IPMN of the Pancreas. Pancreatology (2017) 17:738-53. doi: 10.1016/j.pan.2017.07.007

93. Carr RA, Yip-Schneider MT, Dolejs S, Hancock BA, Wu H, Radovich M, et al. Pancreatic Cyst Fluid Vascular Endothelial Growth Factor a and Carcinoembryonic Antigen: A Highly Accurate Test for the Diagnosis of Serous Cystic Neoplasm. J Am Coll Surg (2017) S1072-7515(17):30452-0. doi: 10.1016/j.jamcollsurg.2017.05.003

94. Gbormittah FO, Haab BB, Partyka K, Garcia-Ott C, Hancapie M, Hancock WS. Characterization of Glycoproteins in Pancreatic Cyst Fluid Using a HighPerformance Multiple Lectin Affinity Chromatography Platform. J Proteome Res (2014) 13:289-99. doi: 10.1021/pr400813u

95. Mohr VH, Vortmeyer AO, Zhuang Z, Libutti SK, Walther MM, Choyke PL, et al. Histopathology and Molecular Genetics of Multiple Cysts and Microcystic (Serous) Adenomas of the Pancreas in Von Hippel-Lindau Patients. Am J Pathol (2000) 157:1615-21. doi: 10.1016/S0002-9440(10) 64799-2

96. Basturk O, Coban I, Adsay NV. Pancreatic Cysts: Pathologic Classification, Differential Diagnosis, and Clinical Implications. Arch Pathol Lab Med (2009) 133:423-38. doi: 10.5858/133.3.423

97. Baiocchi GL, Molfino S, Frittoli B, Pigozzi G, Gheza F, Gaverini G, et al. Increased Risk of Second Malignancy in Pancreatic Intraductal Papillary Mucinous Tumors: Review of the Literature. World J Gastroenterol (2015) 21:7313-9. doi: 10.3748/wjg.v21.i23.7313

98. Chiaro MD, Besselink MG, Scholten L, Bruno MJ, Cahen GL, Gress TM, et al. European Evidence-Based Guidelines on Pancreatic Cystic Neoplasms. Gut (2018) 67:789-804. doi: 10.1136/gutjnl-2018-316027

\section{Conflict of Interest: Author PW was employed by GE Healthcare.}

The remaining authors declare that the research was conducted in the absence of any commercial or financial relationships that could be construed as a potential conflict of interest.

Publisher's Note: All claims expressed in this article are solely those of the authors and do not necessarily represent those of their affiliated organizations, or those of the publisher, the editors and the reviewers. Any product that may be evaluated in this article, or claim that may be made by its manufacturer, is not guaranteed or endorsed by the publisher.

Copyright (C) $2022 \mathrm{Hu}, \mathrm{Hu}$, Wang, Ma, Yue, Tang, Liu, Wu, Peng and Tong. This is an open-access article distributed under the terms of the Creative Commons Attribution License (CC BY). The use, distribution or reproduction in other forums is permitted, provided the original author(s) and the copyright owner(s) are credited and that the original publication in this journal is cited, in accordance with accepted academic practice. No use, distribution or reproduction is permitted which does not comply with these terms. 